\title{
On the Motion of Harmonically Excited Spring Pendulum in Elliptic Path Near Resonances
}

\author{
T. S. Amer, ${ }^{1}$ M. A. Bek, ${ }^{2}$ and I. S. Hamada ${ }^{1}$ \\ ${ }^{1}$ Department of Mathematics, Faculty of Science, Tanta University, Tanta 31527, Egypt \\ ${ }^{2}$ Department of Physics and Engineering Mathematics, Faculty of Engineering, Tanta University, Tanta 31734, Egypt
}

Correspondence should be addressed to T. S. Amer; tarek.amer@science.tanta.edu.eg

Received 30 June 2016; Revised 26 September 2016; Accepted 4 October 2016

Academic Editor: Ciprian G. Gal

Copyright (C) 2016 T. S. Amer et al. This is an open access article distributed under the Creative Commons Attribution License, which permits unrestricted use, distribution, and reproduction in any medium, provided the original work is properly cited.

\begin{abstract}
The response of a nonlinear multidegrees of freedom (M-DOF) for a nature dynamical system represented by a spring pendulum which moves in an elliptic path is investigated. Lagrange's equations are used in order to derive the governing equations of motion. One of the important perturbation techniques MS (multiple scales) is utilized to achieve the approximate analytical solutions of these equations and to identify the resonances of the system. Besides, the amplitude and the phase variables are renowned to study the steady-state solutions and to recognize their stability conditions. The time history for the attained solutions and the projections of the phase plane are presented to interpret the behavior of the dynamical system. The mentioned model is considered one of the important scientific applications like in instrumentation, addressing the oscillations occurring in sawing buildings and the most of various applications of pendulum dampers.
\end{abstract}

\section{Introduction}

Dynamical system is considered a collection of particles in motion with finite numbers of DOF and can be determined through some processes during a period of time. Chaos theory studies the behavior of dynamical systems that are oversensitive to initial conditions and is considered one of the most important subjects in applied mathematics, physics, and engineering fields. It has several applications ranging from weather forecasts, technology, and physical and life sciences. The kinematical nonlinear systems are of great interest for many outstanding researchers during the last three decades. In $[1,2]$, the dynamical behavior of such systems is investigated as good models in applied mechanics. Tousi and Bajaj in [3] studied period doubling bifurcations and modulated motion in 2-DOF of a nonlinear system. Moreover, Maewal in [4] examined chaos of the forced response of an excited elastic beam through the numerical solutions of the governing system of his suggested model. In [5], the author used the MS method to construct the expansion of the parametric excitation of two internally resonant oscillators up to the first order. The author determined the solutions of the steadystate case and checked the corresponding stability. In [6], the authors transformed the governing system of the excited buckled beam to approximate one and examined the stability of the equilibrium solutions to obtain Hopf bifurcations and a sequence of period doubling one, leading to chaotic motion. The response of an excited weakly vibrating system with 2 DOF close to resonance is investigated in [7]. The response of the considered system is checked using the averaging method and the numerical integration. In [8], Lee and Park investigated the excitation of spring pendulum using the MS technique and showed that the obtained approximate autonomous system has bifurcations. The effect of the higherorder expansions of this problem with internal resonance was examined in [9]. In [10], the authors investigated the fourthorder approximate solution for the governing system and the stability of such system of a similar problem when the stiffness of the spring becomes nonlinear. In [11], the authors investigated the same problem besides any equilibrium state using MS method and they also examined the general manner of the system through utilizing the basin boundaries of 
attractors. They observed that these boundaries may be fractal and the damping coefficients have a great effect during the chaotic motion. The problem of the nonlinear behavior of a 2DOF oscillating system coupled with nonlinear damper and nonlinear spring is studied in [12]. The authors showed that, according to certain values for damping and stiffness coefficients, the oscillating amplitude for the examined model is reduced to give better design for the nonlinear absorber. In [13], Amer and Bek studied the response of the excited elastic pendulum in which the motion of the suspended point is considered in a circular path. The governing nonautonomous system is transformed to autonomous ones up to third order using the MS method. They found that the resulted system has bifurcations leading to chaos. Another pendulum model is examined in [14] when the suspended point moves in a prescribed path. The governing equations of motion have been obtained and solved analytically using the multiple scales method. The authors concentrated on predicting the resonance conditions and they studied these cases. This problem was generalized in [15] to study the vibrations of a rigid body as a pendulum model. Many interested examples for the motion of nonlinear systems can be found in [16$20]$ and the references herein. However, in [17] the authors investigated the motion of a spring pendulum which is moving in circular path. In $[18,19]$ they generalized their previous work by considering the effect of a damper to the motion.

In the current work, we extend the previous work in [14] for the nonlinear behavior of kinematically excited spring pendulum in which its suspension point moves in an elliptic path. Our main aim is to reveal the resonances conditions and to outline on a case of simultaneously resonances. Among the perturbation methods, the MS method is used to obtain the modulation equations determining all possible steadystate solutions. The graphical representations for suggested physical parameters of the obtained steady-state solutions are presented. Some examples are given as limit cases from the motion of considered model in order to simulate the dynamical behavior of this model.

\section{Description of the Problem}

Let us consider a dynamical system which consists of a mass $m$, suspended from one end of a massless linear spring having stiffness $\kappa$ and statically stretched length $\ell$. The other end is attached to the point $O_{1}$ that moves in an elliptical path in which $2 a$ and $2 b$ represent the lengths of the minor and the major axes of the ellipse, respectively; see Figure 1. Take into account that the corresponding point of $Q$ (located on the auxiliary circle $b$ ) is the point $O_{1}$ (located on the ellipse) and this point moves with angular velocity $\Omega$. Let us denote the horizontal axis by $O Y$ and the downward one by $O X$, in which both of them have the same origin $O$ of the ellipse. Let $M(t)$ be a moment acts about the point $O_{1}$ in a anticlockwise direction, $F(t)$ indicates the acting force on the mass $m$ in the elongation's direction of the spring, and $F_{r}$ refers to the damping force acts on $m$ along the pendulum length.

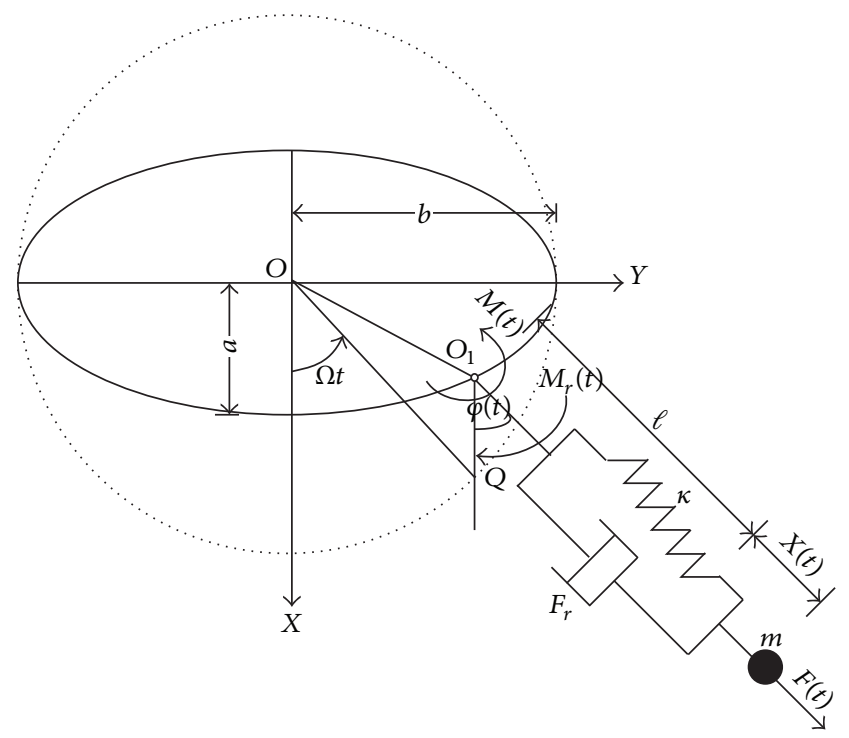

Figure 1: Description of the motion.

To gain over the governing system of motion, we consider the planar motion of our model. So after time $t$ the coordinates of the suspended point $O_{1}\left(x_{O_{1}}, y_{O_{1}}\right)$ can be expressed as

$$
\begin{aligned}
& x_{\mathrm{O}_{1}}=a \cos (\Omega t), \\
& y_{\mathrm{O}_{1}}=b \sin (\Omega t) .
\end{aligned}
$$

Use the following Lagrange's equations of the second type to obtain the equations of motion:

$$
\begin{gathered}
\frac{d}{d t}\left(\frac{\partial L}{\partial \dot{X}}\right)-\left(\frac{\partial L}{\partial X}\right)=Q_{X}, \\
\frac{d}{d t}\left(\frac{\partial L}{\partial \dot{\varphi}}\right)-\left(\frac{\partial L}{\partial \varphi}\right)=Q_{\varphi}
\end{gathered}
$$

where $L=T-V$ expresses Lagrange's function and $(X, \varphi)$ and $(\dot{X}, \dot{\varphi})$ represent the generalized coordinates and velocities of the mentioned model, respectively. The potential and kinetic energies of the considered system are given, respectively, as

$$
\begin{aligned}
V= & \frac{1}{2} \kappa\left(\frac{m g}{\kappa}+X\right)^{2}-m g[a \cos \Omega t+(\ell+X) \cos \varphi], \\
T= & \frac{m}{2}\left[\Omega^{2}\left(a^{2} \sin ^{2} \Omega t+b^{2} \cos ^{2} \Omega t\right)\right] \\
& +m \Omega \dot{X}(b \cos \Omega t \sin \varphi-a \sin \Omega t \cos \varphi) \\
& +m \Omega(\ell+X)(b \cos \Omega t \cos \varphi+a \sin \Omega t \sin \varphi) \dot{\varphi} \\
& +\frac{m}{2}(\ell+X)^{2} \dot{\varphi}^{2}+\frac{m}{2} \dot{X}^{2} .
\end{aligned}
$$

Here, $g$ denotes the gravity acceleration, $X$ is the spring elongation, and $\varphi$ is the angle between the vertical and line directed through the mass $m$.

In addition to the influence of the kinematic excitation on the examined system, the moments $M(t)=M_{0} \cos \left(\Omega_{2} t\right)$ and 
$M_{r}(t)=B_{2} \dot{\varphi}$ (of the linear viscous damping) have an extra effect around $O$. Furthermore, the force $F(t)=F_{0} \cos \left(\Omega_{1} t\right)$ and the linear viscous damping $F_{r}=B_{1} \dot{X}$ are acting on the mass $m$ and directed along the pendulum arm in which $B_{1}$ and $B_{2}$ denote the viscous coefficients.

According to the nonconservative forces, the generalized forces take the form

$$
\begin{aligned}
& Q_{X}=F_{0} \cos \left(\Omega_{1} t\right)-B_{1} \dot{X}, \\
& Q_{\varphi}=M_{0} \cos \left(\Omega_{2} t\right)-B_{2} \dot{\varphi},
\end{aligned}
$$

where $\Omega_{1}$ and $\Omega_{2}$ are forcing frequencies of $F(t)$ and $M(t)$, respectively.

Substituting (3) and (4) into (2), we obtain easily the governing system of motion as

$$
\begin{aligned}
& \ddot{x}+c_{1} \dot{x}-(1+x) \dot{\varphi}^{2}+\omega_{1}^{2} x+\omega_{2}^{2}(1-\cos \varphi) \\
& -\frac{\Omega^{2}}{\ell}(a \cos \Omega t \cos \varphi+b \sin \Omega t \sin \varphi)=f_{1} \cos \Omega_{1} t, \\
& (1+x)^{2} \ddot{\varphi}+c_{2} \dot{\varphi}+2(1+x) \dot{x} \dot{\varphi}+\omega_{2}^{2}(1+x) \sin \varphi \\
& +\frac{\Omega^{2}}{\ell}(a \cos \Omega t \sin \varphi-b \sin \Omega t \cos \varphi)(1+x) \\
& =f_{2} \cos \Omega_{2} t
\end{aligned}
$$

where

$$
\begin{aligned}
x & =\frac{X}{\ell}, \\
\omega_{1}^{2} & =\frac{\kappa}{m}, \\
\omega_{2}^{2} & =\frac{g}{\ell}, \\
c_{1} & =\frac{B_{1}}{m}, \\
c_{2} & =\frac{B_{2}}{m \ell^{2}}, \\
f_{1} & =\frac{F_{0}}{m \ell}, \\
f_{2} & =\frac{M_{0}}{m \ell^{2}} .
\end{aligned}
$$

\section{The Analytical Solution}

The aim of this section is obtain the analytic solutions of the previous equations of motion using the MS method and to get the modulation equations in order to obtain the resonances conditions. So it is necessary to start with the approximations of $\sin \varphi$ and $\cos \varphi$ included in (5) using Taylor series up to the second terms as

$$
\begin{aligned}
& \sin \varphi \cong \varphi-\frac{1}{6} \varphi^{3}, \\
& \cos \varphi \cong 1-\frac{1}{2} \varphi^{2} .
\end{aligned}
$$

It should be noticed that this approximation is valid in a neighborhood of all positions of static equilibrium.

It is customary to define both of the damping coefficients and the amplitudes of external forces, in order to achieve the analysis procedure as

$$
\begin{aligned}
c_{i} & =\varepsilon^{2} \widetilde{c}_{i}, \\
f_{i} & =\varepsilon^{3} \widetilde{f}_{i}, \\
& \quad i=1,2,
\end{aligned}
$$

in which $\varepsilon$ represents a small parameter. Furthermore, let us assume

$$
\begin{aligned}
& a=\varepsilon^{2} \widetilde{a}, \\
& b=\varepsilon^{2} \widetilde{b} .
\end{aligned}
$$

In which the parameters $\widetilde{f}_{i}, \widetilde{c}_{i}, \tilde{a}, \widetilde{b}$ included in the previous two equalities are of order 1 . Let us introduce the following form for the amplitudes of vibrations:

$$
\begin{aligned}
x & =\varepsilon \tilde{x}, \\
\varphi & =\varepsilon \widetilde{\varphi} .
\end{aligned}
$$

It is important to notice that these amplitudes are of order $\varepsilon$. So we seek the forms of the functions $\widetilde{x}$ and $\tilde{\varphi}$ as

$$
\begin{aligned}
& \tilde{x}(t ; \varepsilon)=\sum_{k=1}^{3} \varepsilon^{k} \widetilde{x}_{k}\left(T_{0}, T_{1}, T_{2}\right)+O\left(\varepsilon^{4}\right), \\
& \tilde{\varphi}(t ; \varepsilon)=\sum_{k=1}^{3} \varepsilon^{k} \widetilde{\varphi}_{k}\left(T_{0}, T_{1}, T_{2}\right)+O\left(\varepsilon^{4}\right),
\end{aligned}
$$

where $T_{n}=\varepsilon^{n} t ; n=0,1,2$ represent the independent variables of the different time scales. Also, the differentiations are transformed into the forms

$$
\begin{aligned}
\frac{d}{d t}= & \frac{\partial}{\partial T_{0}}+\varepsilon \frac{\partial}{\partial T_{1}}+\varepsilon^{2} \frac{\partial}{\partial T_{2}} \\
\frac{d^{2}}{d t^{2}}= & \frac{\partial^{2}}{\partial T_{0}^{2}}+2 \varepsilon \frac{\partial^{2}}{\partial T_{0} T_{1}}+\varepsilon^{2}\left(\frac{\partial^{2}}{\partial T_{1}^{2}}+2 \frac{\partial^{2}}{\partial T_{0} T_{2}}\right) \\
& +O\left(\varepsilon^{3}\right) .
\end{aligned}
$$

Substituting (7)-(11) into (5), using the operators (12), and then equating the coefficients of like powers of $\varepsilon$ in both sides, 
one obtains the next sets of partial differential equations from second order.

Order of $\varepsilon$

$$
\begin{aligned}
& \frac{\partial^{2} \widetilde{x}_{1}}{\partial T_{0}^{2}}+\omega_{1}^{2} \widetilde{x}_{1}=0, \\
& \frac{\partial^{2} \widetilde{\varphi}_{1}}{\partial T_{0}^{2}}+\omega_{2}^{2} \widetilde{\varphi}_{1}=0 .
\end{aligned}
$$

Order of $\varepsilon^{2}$

$$
\begin{aligned}
\frac{\partial^{2} \tilde{x}_{2}}{\partial T_{0}^{2}}+\omega_{1}^{2} \tilde{x}_{2}= & \left(\frac{\tilde{a} \Omega^{2}}{\ell}\right) \cos \Omega T_{0}-\frac{1}{2} \omega_{2}^{2} \widetilde{\varphi}_{1}^{2}-2 \frac{\partial^{2} \tilde{x}_{1}}{\partial T_{0} T_{1}} \\
& +\left(\frac{\partial \widetilde{\varphi}_{1}}{\partial T_{0}}\right)^{2} \\
\frac{\partial^{2} \widetilde{\varphi}_{2}}{\partial T_{0}^{2}}+\omega_{2}^{2} \widetilde{\varphi}_{2}= & \left(\frac{\widetilde{b} \Omega^{2}}{\ell}\right) \sin \Omega T_{0}-\omega_{2}^{2} \widetilde{x}_{1} \widetilde{\varphi}_{1} \\
& -2 \frac{\partial \widetilde{x}_{1}}{\partial T_{0}} \frac{\partial \widetilde{\varphi}_{1}}{\partial T_{0}}-2 \widetilde{x}_{1} \frac{\partial^{2} \widetilde{\varphi}_{1}}{\partial T_{0}^{2}} \\
& -2 \frac{\partial^{2} \widetilde{\varphi}_{1}}{\partial T_{0} \partial T_{1}} .
\end{aligned}
$$

Order of $\varepsilon^{3}$

$$
\begin{aligned}
& \frac{\partial^{2} \tilde{x}_{3}}{\partial T_{0}^{2}}+\omega_{1}^{2} \widetilde{x}_{3}=\widetilde{f}_{1} \cos \Omega_{1} T_{0}+\frac{\Omega^{2}}{\ell} \widetilde{\varphi}_{1} \widetilde{b} \sin \Omega T_{0} \\
& -\omega_{2}^{2} \widetilde{\varphi}_{1} \widetilde{\varphi}_{2}-\frac{\partial^{2} \widetilde{x}_{1}}{\partial T_{1}^{2}}-\widetilde{c}_{1} \frac{\partial \widetilde{x}_{1}}{\partial T_{0}} \\
& +2 \frac{\partial \widetilde{\varphi}_{1}}{\partial T_{1}} \frac{\partial \widetilde{\varphi}_{1}}{\partial T_{0}}+\widetilde{x}_{1}\left(\frac{\partial \widetilde{\varphi}_{1}}{\partial T_{0}}\right)^{2} \\
& +2 \frac{\partial \widetilde{\varphi}_{1}}{\partial T_{0}} \frac{\partial \widetilde{\varphi}_{2}}{\partial T_{0}}-2 \frac{\partial^{2} \widetilde{x}_{1}}{\partial T_{0} \partial T_{2}} \\
& -2 \frac{\partial^{2} \tilde{x}_{2}}{\partial T_{0} \partial T_{1}} \\
& \frac{\partial^{2} \widetilde{\varphi}_{3}}{\partial T_{0}^{2}}+\omega_{2}^{2} \widetilde{\varphi}_{3}=\widetilde{f}_{2} \cos \Omega_{2} T_{0}+\frac{\tilde{b} \Omega^{2}}{\ell} \tilde{x}_{1} \sin \Omega T_{0} \\
& -\frac{\widetilde{a} \Omega^{2}}{\ell} \widetilde{\varphi}_{1} \cos \Omega T_{0}-\omega_{2}^{2} \widetilde{x}_{2} \widetilde{\varphi}_{1} \\
& -\omega_{2}^{2} \widetilde{x}_{1} \widetilde{\varphi}_{2}-\frac{\partial^{2} \widetilde{\varphi}_{1}}{\partial T_{1}^{2}}-2 \frac{\partial \widetilde{x}_{1}}{\partial T_{0}} \frac{\partial \widetilde{\varphi}_{1}}{\partial T_{1}} \\
& -\widetilde{c}_{2} \frac{\partial \widetilde{\varphi}_{1}}{\partial T_{0}}-2 \frac{\partial \widetilde{x}_{1}}{\partial T_{1}} \frac{\partial \widetilde{\varphi}_{1}}{\partial T_{0}}-2 \frac{\partial \widetilde{x}_{2}}{\partial T_{0}} \frac{\partial \widetilde{\varphi}_{1}}{\partial T_{0}} \\
& -2 \frac{\partial \widetilde{x}_{1}}{\partial T_{0}} \frac{\partial \widetilde{\varphi}_{2}}{\partial T_{0}}-2 \frac{\partial^{2} \widetilde{\varphi}_{1}}{\partial T_{0} \partial T_{2}}
\end{aligned}
$$

$$
\begin{aligned}
& -4 \tilde{x}_{1} \frac{\partial^{2} \widetilde{\varphi}_{1}}{\partial T_{0} \partial T_{1}}-2 \frac{\partial^{2} \widetilde{\varphi}_{2}}{\partial T_{0} \partial T_{1}} \\
& -\widetilde{x}_{1}^{2} \frac{\partial^{2} \widetilde{\varphi}_{1}}{\partial T_{0}^{2}}-2 \widetilde{x}_{2} \frac{\partial^{2} \widetilde{\varphi}_{1}}{\partial T_{0}^{2}}-2 \widetilde{x}_{1} \frac{\partial^{2} \widetilde{\varphi}_{2}}{\partial T_{0}^{2}} \\
& -2 \widetilde{x}_{1} \frac{\partial \tilde{x}_{1}}{\partial T_{0}} \frac{\partial \widetilde{\varphi}_{1}}{\partial T_{0}}+\frac{\omega_{2}^{2}}{6} \widetilde{\varphi}_{1}^{3} .
\end{aligned}
$$

In the framework of (11), the main system of governing equations (5) is converted into another one which consists of second-order partial differential equations.

The solutions of (13) take the form

$$
\begin{aligned}
& x_{1}=A_{1}\left(T_{1}, T_{2}\right) e^{i \omega_{1} T_{0}}+\bar{A}_{1}\left(T_{1}, T_{2}\right) e^{-i \omega_{1} T_{0}}, \\
& \varphi_{1}=A_{2}\left(T_{1}, T_{2}\right) e^{i \omega_{2} T_{0}}+\bar{A}_{2}\left(T_{1}, T_{2}\right) e^{-i \omega_{2} T_{0}},
\end{aligned}
$$

where $A_{i} ;(i=1,2)$ are unknown complex functions that can be determined and $\bar{A}_{i}$ are the corresponding complex conjugate.

Substituting (16) into (14) and then omitting the terms that produce the secular ones, we get the second-order approximations in the form

$$
\begin{aligned}
x_{2}= & \frac{\omega_{2}^{2}}{\omega_{1}^{2}} A_{2} \bar{A}_{2}-\frac{a \Omega^{2} e^{i \Omega T_{0}}}{2 \ell\left(\Omega^{2}-\omega_{1}^{2}\right)}-\frac{3 \omega_{2}^{2} A_{2}^{2} e^{2 i \omega_{2} T_{0}}}{2\left(\omega_{1}^{2}-4 \omega_{2}^{2}\right)} \\
& + \text { C.C., } \\
\varphi_{2}= & \frac{i b \Omega^{2} e^{i \Omega T_{0}}}{2 \ell\left(\Omega^{2}-\omega_{2}^{2}\right)}-\frac{\omega_{2}\left(2 \omega_{1}+\omega_{2}\right)}{\omega_{1}\left(2 \omega_{2}+\omega_{1}\right)} A_{1} A_{2} e^{i\left(\omega_{1}+\omega_{2}\right) T_{0}} \\
& +\frac{\omega_{2}\left(\omega_{2}-2 \omega_{1}\right)}{\omega_{1}\left(2 \omega_{2}-\omega_{1}\right)} A_{1} \bar{A}_{2} e^{i\left(\omega_{1}-\omega_{2}\right) T_{0}}+\text { C.C., }
\end{aligned}
$$

where C.C. express about the complex conjugates of the preceding terms.

As we developed previously, substitute (16)-(17) into (15) and then remove secular terms to obtain the third-order approximations in the form

$$
\begin{aligned}
x_{3} & =\frac{e^{i \Omega_{1} T_{0}} f_{1}}{2\left(\omega_{1}^{2}-\Omega^{2}\right)}+\frac{i b \Omega^{4} e^{i\left(\Omega+\omega_{2}\right) T_{0}} A_{2}}{2 \ell\left(\Omega^{2}-\omega_{2}^{2}\right)\left[\left(\Omega+\omega_{2}\right)^{2}-\omega_{1}^{2}\right]} \\
& +\frac{i b \Omega^{4} e^{i\left(\Omega-\omega_{2}\right) T_{0}} \bar{A}_{2}}{2 \ell\left(\Omega^{2}-\omega_{2}^{2}\right)\left[\left(\Omega-\omega_{2}\right)^{2}-\omega_{1}^{2}\right]} \\
& -\frac{3 e^{i\left(\omega_{1}+2 \omega_{2}\right) T_{0}} \omega_{2}\left(\omega_{2}+\omega_{1}\right) A_{1} A_{2}^{2}}{4 \omega_{1}\left(\omega_{1}+2 \omega_{2}\right)} \\
& +\frac{3 e^{i\left(\omega_{1}-2 \omega_{2}\right) T_{0}} \omega_{2}\left(\omega_{2}-\omega_{1}\right) A_{1} \bar{A}_{2}^{2}}{4 \omega_{1}\left(2 \omega_{2}-\omega_{1}\right)} \\
& +\frac{2 i b \omega_{2} \Omega^{3} e^{i\left(\Omega+\omega_{2}\right) T_{0}} A_{2}}{2 \ell\left(\Omega^{2}-\omega_{2}^{2}\right)\left[\left(\Omega+\omega_{2}\right)^{2}-\omega_{1}^{2}\right]} \\
& -\frac{2 i b \omega_{2} \Omega^{3} e^{i\left(\Omega-\omega_{2}\right) T_{0}} \bar{A}_{2}}{2 \ell\left(\Omega^{2}-\omega_{2}^{2}\right)\left[\left(\Omega-\omega_{2}\right)^{2}-\omega_{1}^{2}\right]}+\text { C.C., }
\end{aligned}
$$




$$
\begin{aligned}
\varphi_{3} & =\frac{f_{2} e^{i \Omega_{2} T_{0}}}{2\left(\omega_{2}^{2}-\Omega_{2}^{2}\right)}+\frac{i b \Omega^{3} e^{i\left(\omega_{1}+\Omega\right) T_{0}} A_{1}\left(2 \omega_{1}+\Omega\right)}{2 \ell\left(\Omega^{2}-\omega_{2}^{2}\right)\left[\omega_{2}^{2}-\left(\Omega+\omega_{1}^{2}\right)\right]} \\
& +\frac{i b \Omega^{3} e^{i\left(\Omega-\omega_{1}\right) T_{0}} \bar{A}_{1}\left(2 \omega_{1}-\Omega\right)}{2 \ell\left(\Omega^{2}-\omega_{2}^{2}\right)\left[\left(\Omega-\omega_{1}\right)^{2}-\omega_{2}^{2}\right]} \\
& -\frac{e^{i\left(2 \omega_{1}+2 \omega_{2}\right) T_{0}} \omega_{2}\left(11 \omega_{2} \omega_{1}^{2}+6 \omega_{1} \omega_{2}^{2}+\omega_{2}^{3}+6 \omega_{1}^{3}\right) A_{1}^{2} A_{2}}{\omega_{1}\left(2 \omega_{2}+\omega_{1}\right)\left[\omega_{2}^{2}-\left(2 \omega_{1}+\omega_{2}\right)^{2}\right]} \\
& +\frac{e^{i\left(2 \omega_{1}-\omega_{2}\right) T_{0}} \omega_{2}\left(11 \omega_{2} \omega_{1}^{2}-6 \omega_{1} \omega_{2}^{2}+\omega_{2}^{3}-6 \omega_{1}^{3}\right) A_{1}^{2} \bar{A}_{2}}{\omega_{1}\left(2 \omega_{2}-1\right)\left[\omega_{2}^{2}-\left(2 \omega_{1}-\omega_{2}\right)^{2}\right]} \\
& -\frac{e^{3 i \omega_{2} T_{0}}\left(49 \omega_{2}^{2}-\omega_{1}^{2}\right) A_{2}^{3}}{48\left(4 \omega_{2}^{2}-\omega_{1}^{2}\right)}+\text { C.C. }
\end{aligned}
$$

The unknowns $A_{1}$ and $A_{2}$ can be determined from the conditions of removing the secular terms.

\section{Modulation Equations Near Resonances}

The main task of this section is to determine the resonances cases. For this purpose, we note that solutions (17)-(18) have singularities when any of their denominators equals zero. So the secular terms appearing on the right sides of (14)(15) make system (17)-(18) insignificant when some frequency conditions are hold. According to this discussion and to the previous solutions, the resonance cases can be classified as follows:

(i) Principal (primary) external resonances occurring at $\Omega \approx \omega_{1}$ and $\Omega_{2} \approx \omega_{2}$.

(ii) The spring's resonance arising from the kinematic excitation at $\Omega \approx \omega_{1}$.

(iii) The pendulum resonance produced from kinematic excitation at $\Omega \approx \omega_{2}$.

(iv) Internal resonance occurring at $\omega_{1}=2 \omega_{2}$.

(v) Combined resonances at $\Omega= \pm\left(\omega_{1}-\omega_{2}\right)$ and $\Omega=$ $\pm\left(\omega_{1}+\omega_{2}\right)$.

The behavior of the system will be very complicated if the natural frequencies satisfy the above resonance cases. Let us examine the first case of resonance categories $\Omega \approx \omega_{1}$ and $\Omega_{2} \approx \omega_{2}$ appearing simultaneously. Under the present circumstances and in order to investigate the resonances, one inserts the following detuning parameters $\sigma_{1}$ and $\sigma_{2}$ according to

$$
\begin{aligned}
\Omega & =\omega_{1}+\varepsilon \widetilde{\sigma}_{1}, \\
\Omega_{2} & =\omega_{2}+\varepsilon \widetilde{\sigma}_{2},
\end{aligned}
$$

where $\sigma_{i}=\varepsilon \widetilde{\sigma}_{i}(i=1,2)$. This means that the detuning parameters describe the closeness of the excitation frequencies $\Omega$ and $\Omega_{2}$ to $\omega_{1}$ and $\omega_{2}$, respectively.
Substituting (19) into (14)-(15) leads to appearance of secular terms. Their elimination produces the following solvability conditions.

Firstly: Second-Order Equations

$$
\begin{array}{r}
\frac{-a \Omega^{2}}{2 \ell} e^{i T_{1} \widetilde{\sigma}_{1}}+2 i \omega_{1} \frac{\partial A_{1}}{\partial T_{1}}=0, \\
2 i \omega_{2} \frac{\partial A_{2}}{\partial T_{1}}=0,
\end{array}
$$

\section{Secondly: Third Order Equations}

$$
\begin{aligned}
& i \omega_{1} \widetilde{c}_{1} A_{1}+\frac{2 A_{1} A_{2} \bar{A}_{2} \omega_{2}^{2}\left(5 \omega_{1}^{2}-11 \omega_{2}^{2}\right)}{\omega_{1}^{2}-4 \omega_{2}^{2}} \\
& +2 i \omega_{1} \frac{\partial A_{1}}{\partial T_{2}}=0 \\
& -\frac{\widetilde{f}_{2}}{2} e^{i \bar{\sigma}_{2} T_{1}}+i \widetilde{\mathcal{c}}_{2} \omega_{2} A_{2}+\frac{A_{1} \bar{A}_{1} A_{2} \omega_{2}^{2}\left(2 \omega_{1}^{2}+\omega_{2}^{2}\right)}{\omega_{1}\left(2 \omega_{2}+\omega_{1}\right)} \\
& -\frac{A_{2}^{2} \bar{A}_{2} \omega_{2}^{4}\left(11 \omega_{1}^{2}-8 \omega_{2}^{2}\right)}{2 \omega_{1}^{2}\left(\omega_{1}^{2}-4 \omega_{2}^{2}\right)}+2 i \omega_{2} \frac{\partial A_{2}}{\partial T_{2}}=0 .
\end{aligned}
$$

Now, we can determine the unknown functions $A_{1}\left(T_{1}\right.$, $\left.T_{2}\right), \bar{A}_{1}\left(T_{1}, T_{2}\right), A_{2}\left(T_{1}, T_{2}\right)$, and $\bar{A}_{2}\left(T_{1}, T_{2}\right)$ from the previous solvability conditions (20)-(21). It is customary to express these functions in the following polar form:

$$
\begin{aligned}
& A_{1}\left(T_{1}, T_{2}\right) \longrightarrow \frac{\tilde{a}_{1}\left(T_{1}, T_{2}\right)}{2} e^{i \psi_{1}\left(T_{1}, T_{2}\right)}, \\
& A_{2}\left(T_{1}, T_{2}\right) \longrightarrow \frac{\tilde{a}_{2}\left(T_{1}, T_{2}\right)}{2} e^{i \psi_{2}\left(T_{1}, T_{2}\right)},
\end{aligned}
$$

in which $\widetilde{a}_{1}, \widetilde{a}_{2}$ and $\psi_{1}, \psi_{2}$ represent the real functions of the solutions $x$ and $\varphi$ and represent both of amplitudes and phases, respectively.

Insert the following definition of modified phases:

$$
\begin{aligned}
& \theta_{1}\left(T_{1}, T_{2}\right)=\widetilde{\sigma}_{1} T_{1}-\psi_{1}\left(T_{1}, T_{2}\right), \\
& \theta_{2}\left(T_{1}, T_{2}\right)=\widetilde{\sigma}_{2} T_{1}-\psi_{2}\left(T_{1}, T_{2}\right),
\end{aligned}
$$

and then use again operators (12) to transform (20)-(21) from the partial derivative form to the ordinary form as

$$
\begin{aligned}
i \frac{d \widetilde{a}_{1}}{d T} & +\widetilde{a}_{1}\left(-\widetilde{\sigma}_{1}+\frac{d \theta_{1}}{d T}\right) \\
& =-\frac{1}{2} i \widetilde{a}_{1} \widetilde{c}_{1}+\frac{11 \omega_{2}^{2}-5 \omega_{1}^{2}}{4 \omega_{1}\left(\omega_{1}^{2}-4 \omega_{2}^{2}\right)} \omega_{2}^{2} \widetilde{a}_{1} \widetilde{a}_{2}^{2}
\end{aligned}
$$




$$
\begin{gathered}
+\frac{\tilde{a} \Omega^{2}}{2 \ell \omega_{1}}\left(\cos \theta_{1}+i \sin \theta_{1}\right), \\
i \frac{d \widetilde{a}_{2}}{d T}+\widetilde{a}_{2}\left(-\widetilde{\sigma}_{2}+\frac{d \theta_{2}}{d T}\right) \\
=-\frac{1}{2} i \widetilde{a}_{2} \widetilde{c}_{2}-\frac{2 \omega_{1}^{2}+\omega_{2}^{2}}{8 \omega_{1}\left(2 \omega_{2}+\omega_{1}\right)} \omega_{2} \widetilde{a}_{2} \tilde{a}_{1}^{2} \\
+\frac{11 \omega_{1}^{2}-8 \omega_{2}^{2}}{16 \omega_{1}^{2}\left(\omega_{1}^{2}-4 \omega_{2}^{2}\right)} \omega_{2}^{3} \widetilde{a}_{2}^{3} \\
+\frac{\widetilde{f}_{2}}{2 \omega_{2}}\left(\cos \theta_{2}+i \sin \theta_{2}\right) .
\end{gathered}
$$

It is worthwhile to notice that definition (23) has a great advantage form due to the fact that the above modulation system is autonomous. Equating the real and imaginary parts in (24) and omitting the symbol for simplicity, then we have a system consisting of four first-order differential equations in the form

$$
\begin{aligned}
a_{1} \frac{d \theta_{1}}{d T}= & a_{1} \sigma_{1}+\frac{11 \omega_{2}^{2}-5 \omega_{1}^{2}}{4 \omega_{1}\left(\omega_{1}^{2}-4 \omega_{2}^{2}\right)} \omega_{2}^{2} a_{1} a_{2}^{2} \\
& +\frac{a\left(\omega_{1}+\sigma_{1}\right)^{2}}{2 \ell \omega_{1}} \cos \theta_{1}, \\
\frac{d a_{1}}{d T}= & -\frac{1}{2} a_{1} c_{1}+\frac{a\left(\omega_{1}+\sigma_{1}\right)^{2}}{2 \ell \omega_{1}} \sin \theta_{1}, \\
a_{2} \frac{d \theta_{2}}{d T}= & a_{2} \sigma_{2}-\frac{2 \omega_{1}^{2}+\omega_{2}^{2}}{8 \omega_{1}\left(2 \omega_{2}+\omega_{1}\right)} \omega_{2} a_{2} a_{1}^{2} \\
& +\frac{11 \omega_{1}^{2}-8 \omega_{2}^{2}}{16 \omega_{1}^{2}\left(\omega_{1}^{2}-4 \omega_{2}^{2}\right)} \omega_{2}^{3} a_{2}^{3}+\frac{f_{2}}{2 \omega_{2}} \cos \theta_{2}, \\
\frac{d a_{2}}{d T}= & -\frac{1}{2} a_{2} c_{2}+\frac{f_{2}}{2 \omega_{2}} \sin \theta_{2} .
\end{aligned}
$$

Moreover, the previous system of (25) characterizes the amplitudes $a_{1}$ and $a_{2}$ and the modified phases $\theta_{1}$ and $\theta_{2}$ for the examined simultaneously resonances cases.

The graphical representations of the numerical solutions for the original system of (5) are presented, after taking into consideration the system of (25), to describe the motion of our dynamical system at any time.

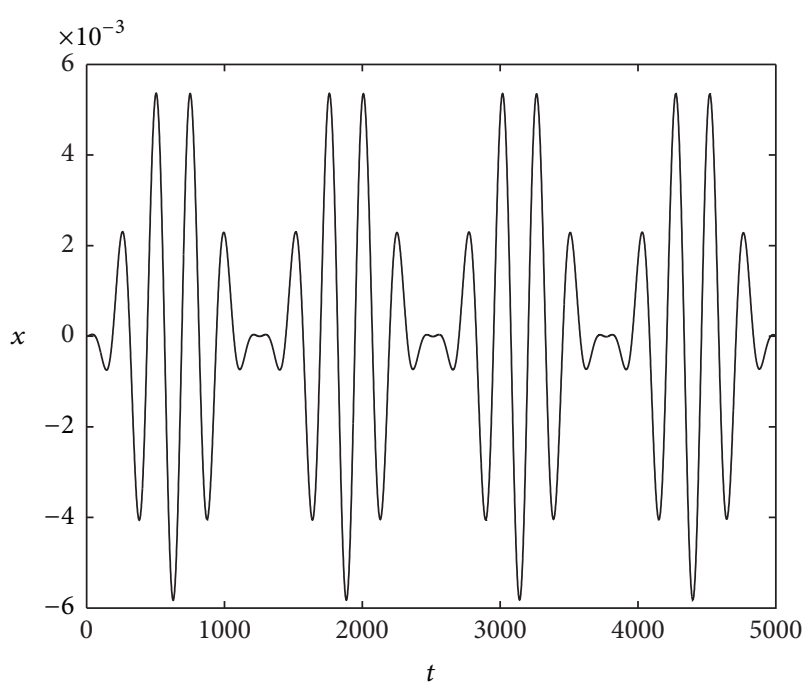

FIgURE 2: Time history for the solution $x$.

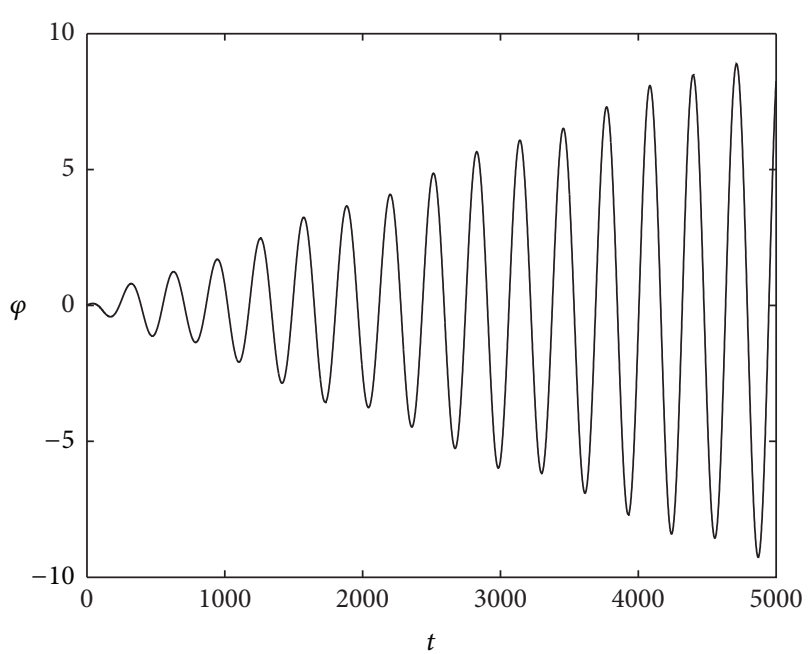

FIgURE 3: Time history for the solution $\varphi$.

Figures 2 and 3 describe the time history of the motion for some selected parameters $c_{1}=c_{2}=0.003\left[\mathrm{~kg} \cdot \mathrm{m}^{2} \cdot \mathrm{s}^{-1}\right], \omega_{1}=$ $\omega_{2}=0.02\left[\mathrm{rad} \cdot \mathrm{s}^{-1}\right], \sigma_{1}=0.01\left[\mathrm{rad} \cdot \mathrm{s}^{-1}\right], \sigma_{2}=0.005\left[\mathrm{rad} \cdot \mathrm{s}^{-1}\right]$, $a=0.3[\mathrm{~m}]$, and $b=0.5[\mathrm{~m}]$. It is not difficult to observe that Figure 2 displays the portrait of the solution $x$ against time $t$ and has the periodic or quasiperiodic behavior when time goes on, while Figure 3 describes the variation of the solution $\varphi$ via $t$ and represents the typical resonance behavior.

\section{Steady-State Solution}

The scope of this section is to obtain the steady-state solutions of both the amplitudes and modified phases corresponding to the zero values of the previous system (25). After omission of the phases $\theta_{1}$ and $\theta_{2}$ from the steady-state solution, the 


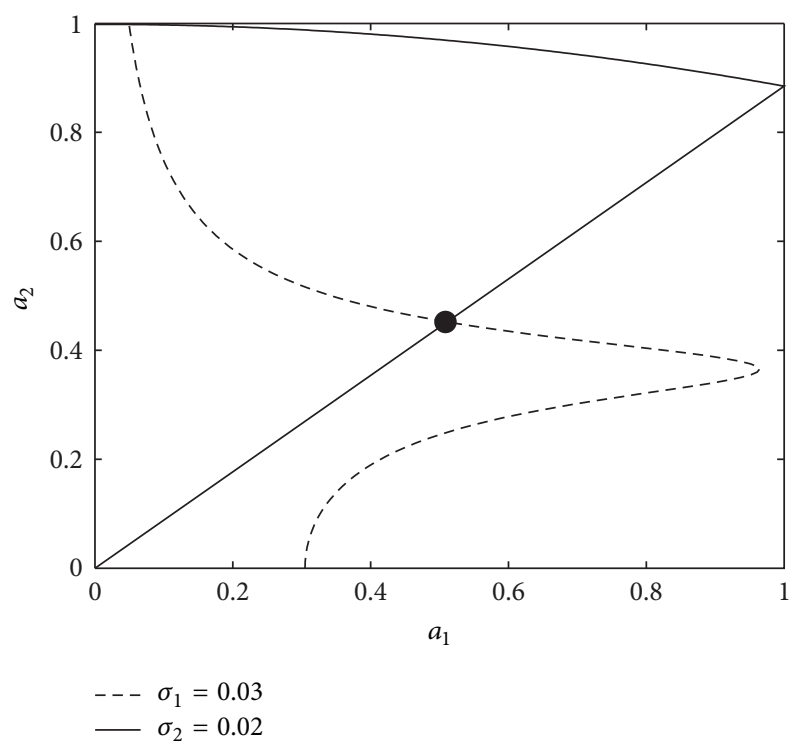

FIGURE 4: Steady-state solution when $\sigma_{1}=0.03\left[\mathrm{rad} \cdot \mathrm{s}^{-1}\right]$ and $\sigma_{2}=$ $0.02\left[\mathrm{rad} \cdot \mathrm{s}^{-1}\right]$.

formulas of frequency response equations take the following form.

For the Case of Parametric Resonance

$$
\begin{gathered}
a_{1}^{2}\left(\frac{\omega_{2}^{2}\left(11 \omega_{2}^{2}-5 \omega_{1}^{2}\right) a_{2}^{2}}{4 \omega_{1}\left(\omega_{1}^{2}-4 \omega_{2}^{2}\right)}+\sigma_{1}\right)^{2}+\frac{c_{1}^{2}}{4} a_{1}^{2} \\
-\frac{a^{2}\left(\omega_{1}+\sigma_{1}\right)^{4}}{4 \ell \omega_{1}^{2}}=0 .
\end{gathered}
$$

For the Case of External Resonance

$$
\begin{aligned}
& a_{2}^{2}\left(-\sigma_{2}+\frac{\left(2 \omega_{1}^{2}+\omega_{2}^{2}\right) \omega_{2} a_{1}^{2}}{8 \omega_{1}\left(2 \omega_{2}+\omega_{1}\right)}\right. \\
& \left.\quad-\frac{\left(11 \omega_{1}^{2}-8 \omega_{2}^{2}\right) \omega_{2}^{3} a_{2}^{2}}{16 \omega_{1}^{2}\left(\omega_{1}^{2}-4 \omega_{2}^{2}\right)}\right)^{2}+\frac{c_{2}^{2}}{4} a_{2}^{2}-\frac{f_{2}^{2}}{4 \omega_{2}^{2}}=0,
\end{aligned}
$$

in which $a_{1}$ and $a_{2}$ represent longitudinal amplitudes and fluctuating vibrations, respectively.

If two resonances occur at the same time, (26) and (27) ought to be a set of nonlinear equations in terms of $a_{1}$ and $a_{2}$. So one can illustrate the possible steady-state solutions close to resonance through a plane which consists of the coordinates $a_{1}$ and $a_{2}$.

The graphs displayed in Figures 4, 5, and 6 show the steady-state solutions of $a_{1}$ via $a_{2}$ when $\sigma_{1}$ and $\sigma_{2}$ take the values $(0.03,0.02)\left[\mathrm{rad} \cdot \mathrm{s}^{-1}\right],(-0.03,-0.03)\left[\mathrm{rad} \cdot \mathrm{s}^{-1}\right]$, and $(0.03,-0.002)\left[\mathrm{rad} \cdot \mathrm{s}^{-1}\right]$, respectively. The dashed lines represent the locus of the roots of (26), and the solid one identifies the solutions of (27). A closer look of these figures carefully shows that the concurrent points of both two curves identify the desired solutions of the system of (26) and (27). These

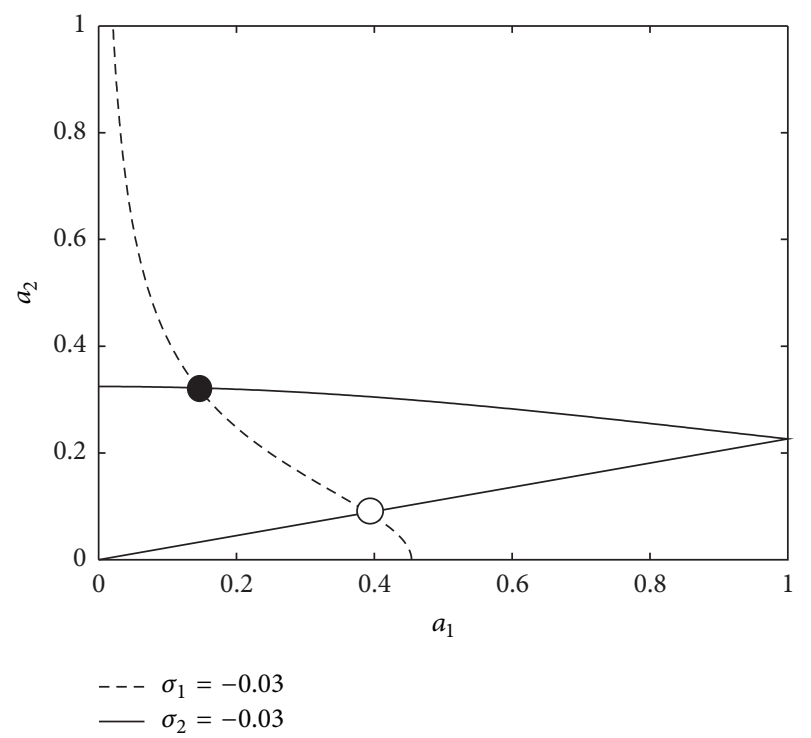

FIGURE 5: Steady-state solution when $\sigma_{1}=\sigma_{2}=-0.03\left[\mathrm{rad} \cdot \mathrm{s}^{-1}\right]$.

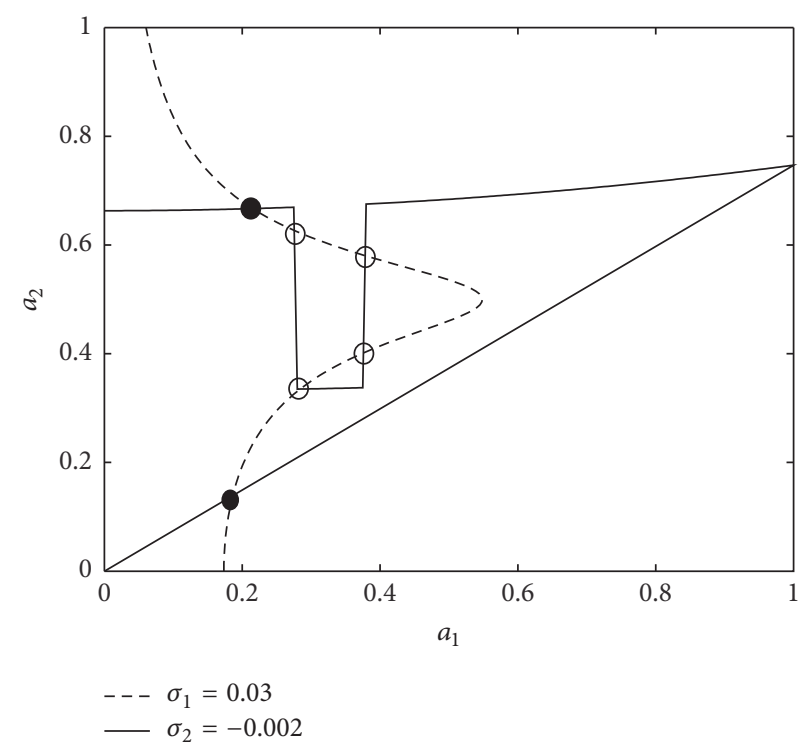

FIGURE 6: Steady-state solution when $\sigma_{1}=0.03\left[\mathrm{rad} \cdot \mathrm{s}^{-1}\right]$ and $\sigma_{2}=$ $-0.002\left[\mathrm{rad} \cdot \mathrm{s}^{-1}\right]$.

points determine definitely the amplitudes of longitudinal and the fluctuating vibrations for the steady state. By the way, the steady states of oscillation may be stable or not. The geometrical representations of the system of (26) and (27) are obtained automatically using MATLAB program after taking into account the following parameters: $\omega_{1}=0.22\left[\mathrm{rad} \cdot \mathrm{s}^{-1}\right]$, $\omega_{2}=0.13\left[\mathrm{rad} \cdot \mathrm{s}^{-1}\right], c_{1}=0.002\left[\mathrm{~kg} \cdot \mathrm{m}^{2} \cdot \mathrm{s}^{-1}\right]$, and $c_{2}=$ $0.003\left[\mathrm{~kg} \cdot \mathrm{m}^{2} \cdot \mathrm{s}^{-1}\right]$.

This elucidates that the desired possible solutions can be expressed as functions of the oscillating system's parameters. Moreover, the lowest number of possible solutions is one and the maximum is may be up to seven. 


\section{Stability Analysis}

One of the important factors for the mentioned problem of the steady-state oscillations is to investigate their stability. For this task, we analyze the manner of the system in a region that is very close to the fixed points.

To discuss the stability for the particular solution of the steady state, we introduce the substitutions

$$
\begin{aligned}
& a_{1}=a_{10}+a_{11}, \\
& a_{2}=a_{20}+a_{21}, \\
& \theta_{1}=\theta_{10}+\theta_{11}, \\
& \theta_{2}=\theta_{20}+\theta_{21}
\end{aligned}
$$

into (25). Here $a_{10}, \theta_{10}, a_{20}$, and $\theta_{20}$ represent the solutions of (25) and $a_{11}, \theta_{11}, a_{21}$, and $\theta_{21}$ denote perturbations which are assumed to be very small, compared to the predecessors. Then the linearized equations take the form

$$
\begin{aligned}
& a_{10} \frac{d \theta_{11}}{d T}=-\frac{a\left(\omega_{1}+\sigma_{1}\right)^{2}}{2 \ell \omega_{1}} \sin \theta_{10} \theta_{11}+\left(\sigma_{1}\right. \\
& \left.+\frac{11 \omega_{2}^{2}-5 \omega_{1}^{2}}{4 \omega_{1}\left(\omega_{1}^{2}-4 \omega_{2}^{2}\right)} \omega_{2}^{2} a_{20}^{2}\right) a_{11}+\frac{11 \omega_{2}^{2}-5 \omega_{1}^{2}}{2 \omega_{1}\left(\omega_{1}^{2}-4 \omega_{2}^{2}\right)} \\
& \cdot a_{10} a_{20} a_{21} \omega_{2}^{2}, \\
& \frac{d a_{11}}{d T}=\frac{a\left(\omega_{1}+\sigma_{1}\right)^{2}}{2 \ell \omega_{1}} \cos \theta_{10} \theta_{11}-\frac{1}{2} a_{11} c_{1}, \\
& a_{20} \frac{d \theta_{21}}{d T}=-\frac{2 \omega_{1}^{2}+\omega_{2}^{2}}{4 \omega_{1}\left(2 \omega_{2}+\omega_{1}\right)} a_{20} a_{10} a_{11} \omega_{2} \\
& +\left(-\frac{2 \omega_{1}^{2}+\omega_{2}^{2}}{8 \omega_{1}\left(2 \omega_{2}+\omega_{1}\right)} a_{10}^{2} \omega_{2}+\sigma_{2}\right. \\
& \left.+\frac{3\left(11 \omega_{1}^{2}-8 \omega_{2}^{2}\right)}{16 \omega_{1}^{2}\left(\omega_{1}^{2}-4 \omega_{2}^{2}\right)} a_{20}^{2} \omega_{2}^{3}\right) a_{21}-\frac{f_{2}}{2 \omega_{2}} \sin \theta_{20} \theta_{21}, \\
& \frac{d a_{21}}{d T}=-\frac{1}{2} a_{21} c_{2}+\frac{f_{2}}{2 \omega_{2}} \cos \theta_{20} \theta_{21} \cdot
\end{aligned}
$$

Take into consideration that the small perturbations $a_{11}$, $\theta_{11}, a_{21}$, and $\theta_{21}$ are unknown functions. Every solution is a linear combination of $C_{i} e^{\lambda T}$, where $C_{i}, i=1,2,3,4$, are constants and $\lambda$ is the eigenvalue corresponding to the unknown perturbation, counted from the real parts of the roots. In this analysis, if the steady-state solutions (fixed points) $a_{10}, \theta_{10}, a_{20}$, and $\theta_{20}$ are asymptotically stable, the real parts of the roots of the following characteristic equation

$$
\lambda^{4}+\Gamma_{1} \lambda^{3}+\Gamma_{2} \lambda^{2}+\Gamma_{3} \lambda+\Gamma_{4}=0
$$

of the set of (29), must be negative. Here $\Gamma_{1}, \Gamma_{2}, \Gamma_{3}$, and $\Gamma_{4}$ take the form

$$
\begin{aligned}
& \Gamma_{1}=\frac{c_{1}}{2}+\frac{c_{2}}{2}+\frac{a\left(\omega_{1}+\sigma_{1}\right)^{2}}{2 \ell \omega_{1}} \sin \theta_{10}+\frac{f_{2}}{2 \omega_{2}} \sin \theta_{20}, \\
& \Gamma_{2}=\frac{c_{1} c_{2}}{4}-a_{20}^{2}\left(\frac{a\left(\omega_{1}+\sigma_{1}\right)^{2}\left(11 \omega_{2}^{2}-5 \omega_{1}^{2}\right)}{8 \ell \omega_{1}^{2}\left(\omega_{1}^{2}-4 \omega_{2}^{2}\right)}\right) \omega_{2}^{2} \cos \theta_{10} \\
& -\frac{a\left(\omega_{1}+\sigma_{1}\right)^{2}}{2 \ell \omega_{1}} \sigma_{1} \cos \theta_{10} \\
& -\frac{f_{2}}{2 \omega_{2}}\left[-a_{10}^{2}\left(\frac{2 \omega_{1}^{2}+\omega_{2}^{2}}{8 \omega_{1}\left(2 \omega_{2}+\omega_{1}\right)}\right) \omega_{2}\right. \\
& \left.+3 a_{20}^{2}\left(\frac{11 \omega_{1}^{2}-8 \omega_{2}^{2}}{16 \omega_{1}^{2}\left(\omega_{1}^{2}-4 \omega_{2}^{2}\right)}\right) \omega_{2}^{3}+\sigma_{2}\right] \cos \theta_{20} \\
& +\frac{1}{2}\left(\frac{a\left(\omega_{1}+\sigma_{1}\right)^{2}}{2 \ell \omega_{1}}\right)\left(c_{1}+c_{2}\right) \sin \theta_{10}+\left(c_{1}+c_{2}\right) \frac{f_{2}}{4 \omega_{2}} \\
& \cdot \sin \theta_{20}+\frac{a\left(\omega_{1}+\sigma_{1}\right)^{2}}{2 \ell \omega_{1}} \frac{f_{2}}{2 \omega_{2}} \sin \theta_{10} \sin \theta_{20} \\
& \Gamma_{3}=-\frac{1}{2} a_{20}^{2} c_{2}\left(\frac{a\left(\omega_{1}+\sigma_{1}\right)^{2}\left(11 \omega_{2}^{2}-5 \omega_{1}^{2}\right)}{8 \ell \omega_{1}^{2}\left(\omega_{1}^{2}-4 \omega_{2}^{2}\right)}\right) \omega_{2}^{2} \cos \theta_{10} \\
& -\frac{1}{2} c_{2}\left(\frac{a\left(\omega_{1}+\sigma_{1}\right)^{2}}{2 \ell \omega_{1}}\right) \sigma_{1} \cos \theta_{10}-\frac{1}{2} c_{1}\left(\frac{f_{2}}{2 \omega_{2}}\right) \\
& \cdot\left[-a_{10}^{2}\left(\frac{2 \omega_{1}^{2}+\omega_{2}^{2}}{8 \omega_{1}\left(2 \omega_{2}+\omega_{1}\right)} \omega_{2}\right)\right. \\
& \left.+3 a_{20}^{2}\left(\frac{11 \omega_{1}^{2}-8 \omega_{2}^{2}}{16 \omega_{1}^{2}\left(\omega_{1}^{2}-4 \omega_{2}^{2}\right)}\right) \omega_{2}^{3}+\sigma_{2}\right] \cos \theta_{20}+\frac{1}{8} \\
& \cdot c_{1} c_{2}\left[\frac{a\left(\omega_{1}+\sigma_{1}\right)^{2}}{\ell \omega_{1}} \sin \theta_{10}+\frac{f_{2}}{\omega_{2}} \sin \theta_{20}\right] \\
& +\frac{1}{2}\left(\frac{a\left(\omega_{1}+\sigma_{1}\right)^{2}}{2 \ell \omega_{1}} \frac{f_{2}}{2 \omega_{2}}\right)\left(c_{1}+c_{2}\right) \sin \theta_{10} \sin \theta_{20} \\
& +\left(\frac{a\left(\omega_{1}+\sigma_{1}\right)^{2}}{2 \ell \omega_{1}} \frac{f_{2}}{2 \omega_{2}}\right)\left(\frac{\left(11 \omega_{2}^{2}-5 \omega_{1}^{2}\right)}{4 \omega_{1}\left(\omega_{1}^{2}-4 \omega_{2}^{2}\right)} \omega_{2}^{2} a_{20}^{2}+\sigma_{1}\right) \\
& \cdot \cos \theta_{10} \sin \theta_{20} \\
& \Gamma_{4}=-4 a_{10}^{2} a_{20}^{2}\left[\frac{a f_{2}\left(\omega_{1}+\sigma_{1}\right)^{2}\left(11 \omega_{2}^{2}-5 \omega_{1}^{2}\right)\left(2 \omega_{1}^{2}+\omega_{2}^{2}\right)}{128 \ell \omega_{1}^{3}\left(\omega_{1}^{2}-4 \omega_{2}^{2}\right)\left(2 \omega_{2}+\omega_{1}\right)}\right] \\
& \cdot \omega_{2}^{2} \cos \theta_{10} \cos \theta_{20}+\left(\frac{a\left(\omega_{1}+\sigma_{1}\right)^{2}}{2 \ell \omega_{1}} \frac{f_{2}}{2 \omega_{2}}\right) \\
& \cdot\left[-a_{10}^{2}\left(\frac{2 \omega_{1}^{2}+\omega_{2}^{2}}{8 \omega_{1}\left(2 \omega_{2}+\omega_{1}\right)}\right) \omega_{2}\right. \\
& \left.+3 a_{20}^{2}\left(\frac{11 \omega_{1}^{2}-8 \omega_{2}^{2}}{16 \omega_{1}^{2}\left(\omega_{1}^{2}-4 \omega_{2}^{2}\right)}\right) \omega_{2}^{3}+\sigma_{2}\right]
\end{aligned}
$$




$$
\begin{aligned}
& \cdot\left(\omega_{2}^{2} a_{20}^{2} \frac{\left(11 \omega_{2}^{2}-5 \omega_{1}^{2}\right)}{4 \omega_{1}\left(\omega_{1}^{2}-4 \omega_{2}^{2}\right)}+\sigma_{1}\right) \cos \theta_{10} \cos \theta_{20}-\frac{1}{2} \\
& \cdot c_{1}\left(\frac{a\left(\omega_{1}+\sigma_{1}\right)^{2}}{2 \ell \omega_{1}} \frac{f_{2}}{2 \omega_{2}}\right)\left[-a_{10}^{2}\left(\frac{2 \omega_{1}^{2}+\omega_{2}^{2}}{8 \omega_{1}\left(2 \omega_{2}+\omega_{1}\right)}\right) \omega_{2}\right. \\
& \left.+3 a_{20}^{2}\left(\frac{11 \omega_{1}^{2}-8 \omega_{2}^{2}}{16 \omega_{1}^{2}\left(\omega_{1}^{2}-4 \omega_{2}^{2}\right)}\right) \omega_{2}^{3}+\sigma_{2}\right] \cos \theta_{20} \sin \theta_{10}-\frac{1}{2} \\
& \cdot c_{2}\left(\frac{a\left(\omega_{1}+\sigma_{1}\right)^{2}}{2 \ell \omega_{1}} \frac{f_{2}}{2 \omega_{2}}\right)\left(\frac{\left(11 \omega_{2}^{2}-5 \omega_{1}^{2}\right)}{4 \omega_{1}\left(\omega_{1}^{2}-4 \omega_{2}^{2}\right)} \omega_{2}^{2} a_{20}^{2}+\sigma_{1}\right) \\
& \cdot \cos \theta_{10} \sin \theta_{20}-\frac{1}{4} c_{1} c_{2}\left(\frac{a\left(\omega_{1}+\sigma_{1}\right)^{2}}{2 \ell \omega_{1}} \frac{f_{2}}{2 \omega_{2}}\right) \sin \theta_{10} \\
& \cdot \sin \theta_{20} .
\end{aligned}
$$

However, according to the Routh-Hurwitz criterion [16], the fundamental conditions of the stability for the particular steady-state solutions will be

$$
\begin{aligned}
& \Gamma_{1}>0, \\
& \Gamma_{3}\left(\Gamma_{1} \Gamma_{2}-\Gamma_{3}\right)-\Gamma_{4} \Gamma_{1}^{2}>0, \\
& \Gamma_{1} \Gamma_{2}-\Gamma_{3}>0, \\
& \Gamma_{4}>0 .
\end{aligned}
$$

The used analysis to check the stability of the proposed solutions that are plotted in Figures 4, 5, and 6 can be interpreted as follows: the points characterized by black dots refer to the stable solutions, and those characterized by hollow circles refer to unstable ones. Figures 7-12 represent the variation of the solution $x$ against the solution $\varphi$ for selected values of the effective parameters $\omega_{2}, \sigma_{1}$, and $\sigma_{2}$ on the motion when $\omega_{1}=0.2\left[\mathrm{rad} \cdot \mathrm{s}^{-1}\right]$.

\section{Examples}

This section is devoted to provide some examples about the motion of the considered model, especially when the axes of the ellipse become equal; that is, $a=b \neq 0$; this elucidates that the motion of the pivot point will be on a circle. Moreover, the supported point becomes fixed; that is, $a=b=0$ (the major and minor axes take the zero value). In addition, the case can be studied when one length of ellipse's axes vanishes ( $a=0$ or $b=0$ ) or more precisely the pivot point moves horizontally or vertically. So let us examine these cases.

Case $1(a=b \neq 0)$. In this case, we observe that the suspended point $O_{1}$ moves in a circular path of radius $a=b=$ $r$. Figure 13 is drawn at $\sigma_{2}=0.005\left[\mathrm{rad} \cdot \mathrm{s}^{-1}\right], \omega_{1}=1\left[\mathrm{rad} \cdot \mathrm{s}^{-1}\right]$, $\omega_{2}=0.5\left[\mathrm{rad} \cdot \mathrm{s}^{-1}\right], r=0.3[\mathrm{~m}]$, and $\ell=1[\mathrm{~m}]$. Parts $(\mathrm{a}, \mathrm{b})$ and (c, d) of this figure portrait the time history of the solutions at $\sigma_{1}=0.01$ and $\sigma_{1}=-0.01$, respectively, while parts (e) and (f) display the projections of the phase planes at the same values of $\sigma_{1}$. Therefore, the present results are in a good agreement with the known previous results as in [17] (in the absence of

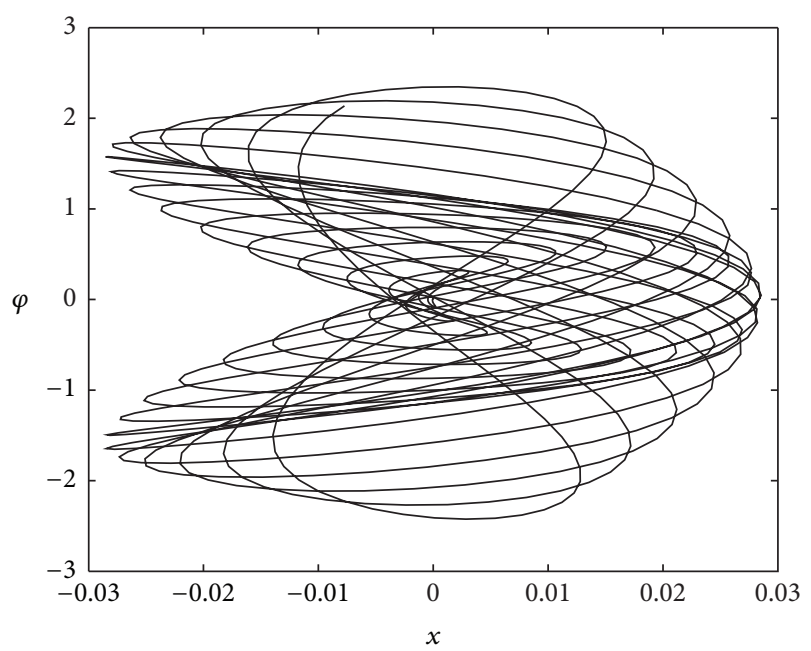

FIgURE 7: Projection on the phase plane when $\omega_{2}=0.1\left[\mathrm{rad} \cdot \mathrm{s}^{-1}\right]$, $\sigma_{1}=0.01\left[\mathrm{rad} \cdot \mathrm{s}^{-1}\right]$, and $\sigma_{2}=0.005\left[\mathrm{rad} \cdot \mathrm{s}^{-1}\right]$.

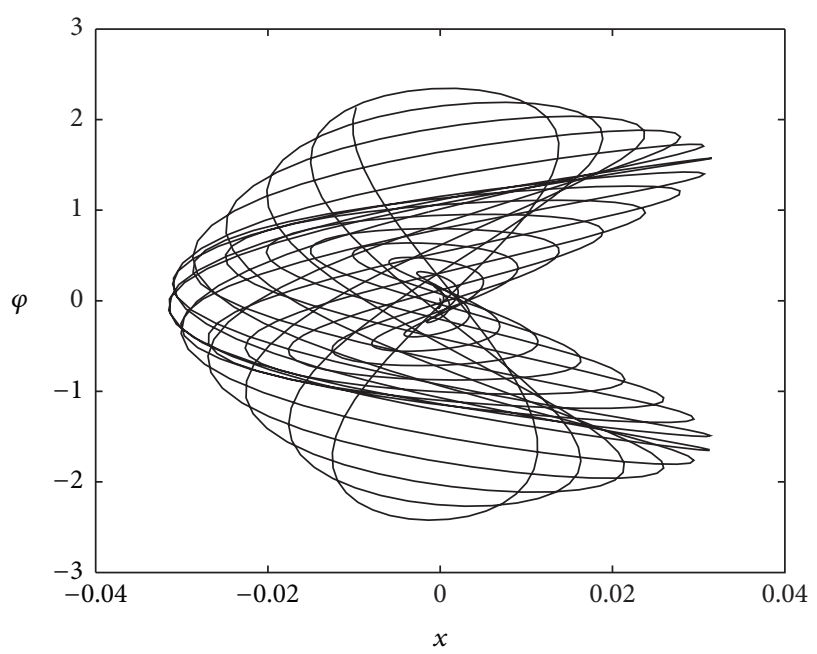

FIGURE 8: Projection on the phase plane when $\omega_{2}=0.1\left[\mathrm{rad} \cdot \mathrm{s}^{-1}\right]$, $\sigma_{1}=-0.01\left[\mathrm{rad} \cdot \mathrm{s}^{-1}\right]$, and $\sigma_{2}=0.005\left[\mathrm{rad} \cdot \mathrm{s}^{-1}\right]$.

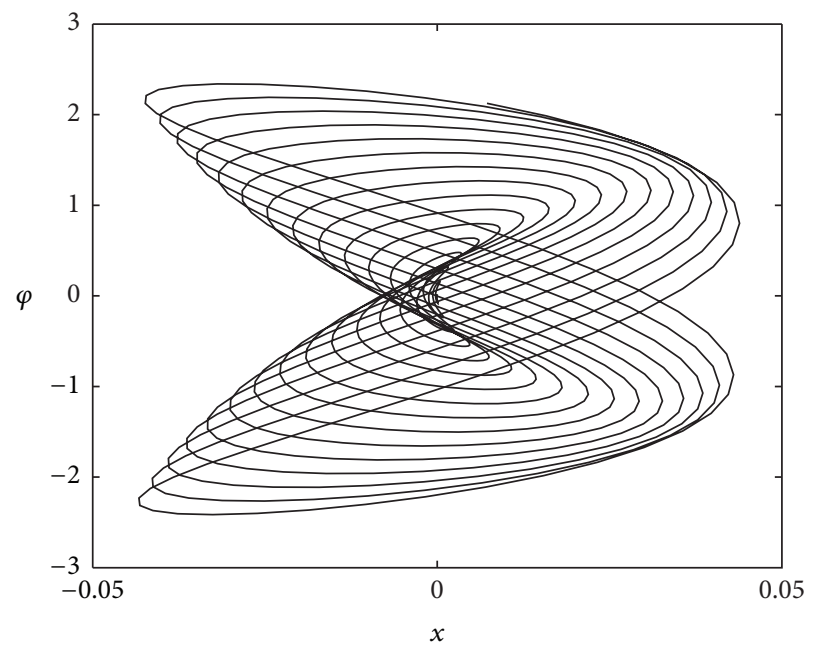

FIGURE 9: Diagram illustrating the variation of $x$ via $\varphi$ when $\omega_{2}=$ $0.1\left[\mathrm{rad} \cdot \mathrm{s}^{-1}\right], \sigma_{1}=0.005\left[\mathrm{rad} \cdot \mathrm{s}^{-1}\right]$, and $\sigma_{2}=0.005\left[\mathrm{rad} \cdot \mathrm{s}^{-1}\right]$. 


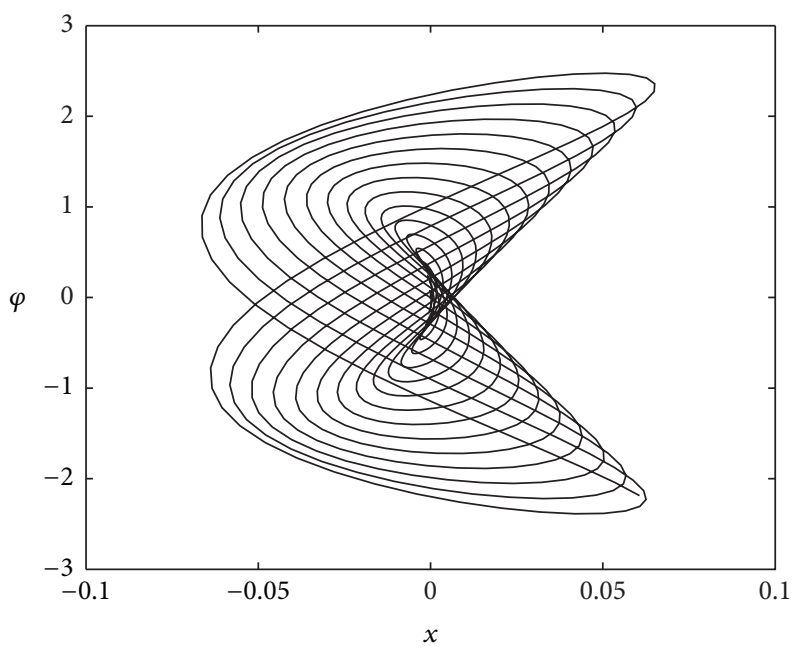

FIGURE 10: Diagram illustrating the variation of $x$ via $\varphi$ when $\omega_{2}=0.1\left[\mathrm{rad} \cdot \mathrm{s}^{-1}\right], \sigma_{1}=-0.005\left[\mathrm{rad} \cdot \mathrm{s}^{-1}\right]$, and $\sigma_{2}=-0.005\left[\mathrm{rad} \cdot \mathrm{s}^{-1}\right]$.

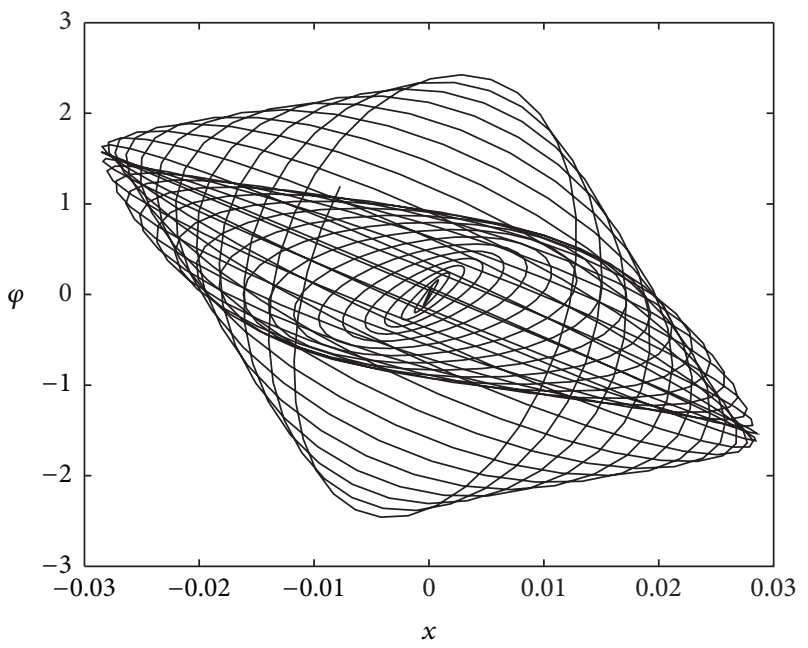

FIGURE 11: Diagram displaying the portrait of $x$ against $\varphi$ when $\omega_{2}=$ $0.2\left[\mathrm{rad} \cdot \mathrm{s}^{-1}\right], \sigma_{1}=0.01\left[\mathrm{rad} \cdot \mathrm{s}^{-1}\right]$, and $\sigma_{2}=0.005\left[\mathrm{rad} \cdot \mathrm{s}^{-1}\right]$.

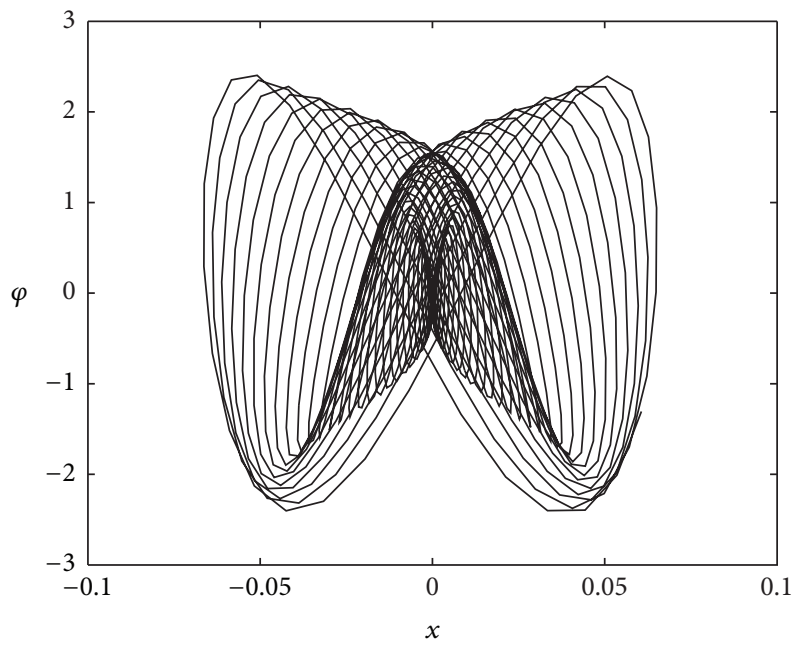

FIGURE 12: Diagram displaying the portrait of $x$ against $\varphi$ when $\omega_{2}=$ $0.4\left[\mathrm{rad} \cdot \mathrm{s}^{-1}\right], \sigma_{1}=-0.005\left[\mathrm{rad} \cdot \mathrm{s}^{-1}\right]$, and $\sigma_{2}=0.005\left[\mathrm{rad} \cdot \mathrm{s}^{-1}\right]$. the damping force $F_{r}$ ) and $[18,19]$ (with the consideration of $\left.F_{r}\right)$.

In this case the modulations of amplitudes for both solutions $x$ and $\varphi$ after a short period of time tend to be more systematic, that is, harmonically, as indicated in parts (a), (b), (c), and (d). On the other hand, the shape of oscillations refers to the effectiveness of nonlinearity. The longitudinal vibration resorts to the sawtooth shape when time goes on till the end of time interval; this is due to the increasing of the nonlinearity parameters. The vibrations of the dynamical model tend to be steady state as shown from the time history plots.

Case $2(a=b=0)$. In this case we shall concentrate on the solutions when the supported point will be fixed. Figure 14 summarizes the results obtained at $\sigma_{2}=0.005\left[\mathrm{rad} \cdot \mathrm{s}^{-1}\right], \omega_{1}=$ $1\left[\mathrm{rad} \cdot \mathrm{s}^{-1}\right], \omega_{2}=0.5\left[\mathrm{rad} \cdot \mathrm{s}^{-1}\right], a=b=0$, and $\ell=1[\mathrm{~m}]$. The effectiveness of increasing time is on the solutions and is illustrated in parts ((a), (b)) and ((c), (d)) of Figure 14 at $\sigma_{1}=0.01\left[\mathrm{rad} \cdot \mathrm{s}^{-1}\right]$ and $\sigma_{1}=-0.01\left[\mathrm{rad} \cdot \mathrm{s}^{-1}\right]$, respectively. The variation of the solution $x$ via solution $\varphi$ is presented in parts (e) and (f) of the same figure. The results of this case are in high consistency with the obtained ones in [20] (in the presence of the linear viscous damping).

Case $3(a=0)$. The task of this case is to interpret the motion of the dynamical system when the pivot point moves horizontally. So the dynamical motion will be in a horizontal axis of length $2 b$ through a long period $[-b, b]$. The graphs displayed in parts (a) and (b) of Figure 15 show the variation of the solutions $x$ and $\varphi$ with the time $t$ when $\sigma_{1}=-0.01\left[\mathrm{rad} \cdot \mathrm{s}^{-1}\right], \sigma_{2}=0.005\left[\mathrm{rad} \cdot \mathrm{s}^{-1}\right], \omega_{1}=1\left[\mathrm{rad} \cdot \mathrm{s}^{-1}\right]$, $\omega_{2}=0.5\left[\mathrm{rad} \cdot \mathrm{s}^{-1}\right]$, and $b=0.5[\mathrm{~m}]$. On the other hand, part (c) of the same figure explicates the variation of $x$ versus $\varphi$ at the same values of $\sigma_{1}$ and $\sigma_{2}$.

Case $4(b=0)$. One of the important concepts is to study the dynamical motion when the supported point $O_{1}$ moves vertically on an axis of length $2 a$ during a long period $[-a, a]$. Several figures have been plotted to illustrate the dynamical 


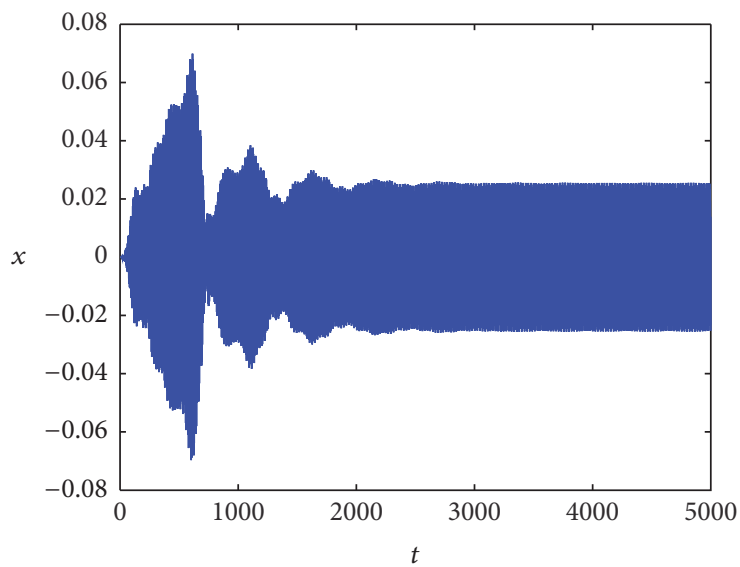

$-\sigma_{1}=0.01, \sigma_{2}=0.005$

(a)

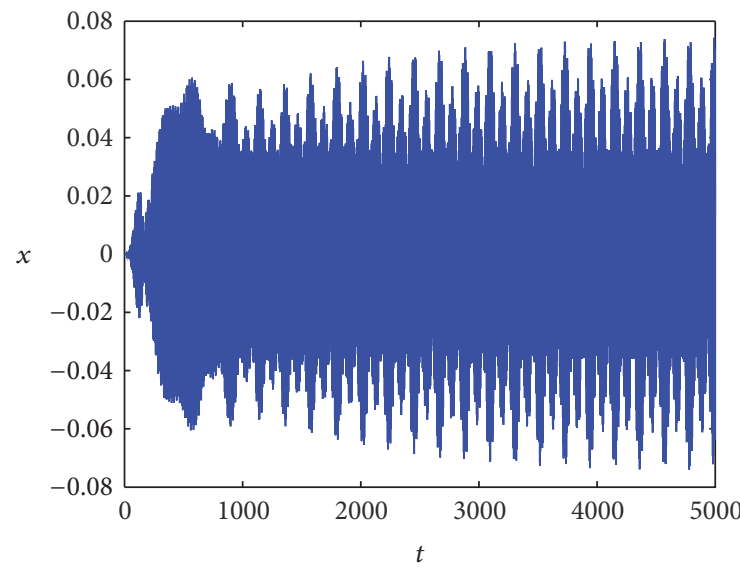

$-\sigma_{1}=-0.01, \sigma_{2}=0.005$

(c)

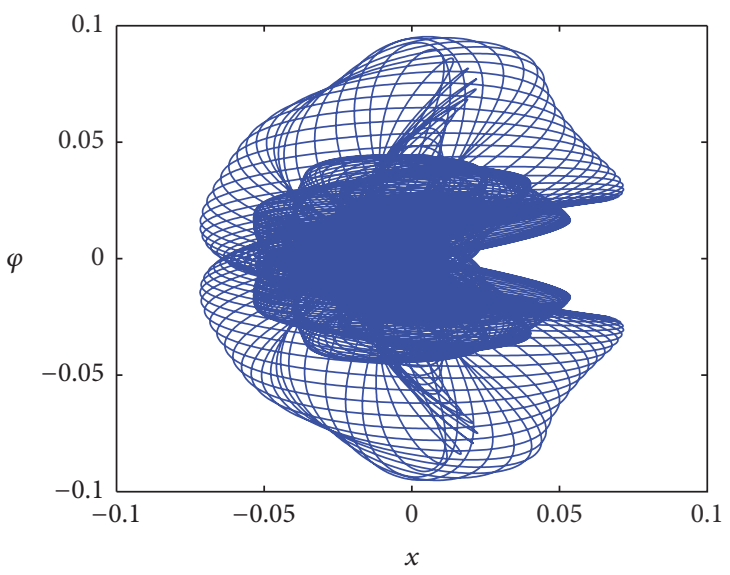

$-\sigma_{1}=0.01, \sigma_{2}=0.005$

(e)

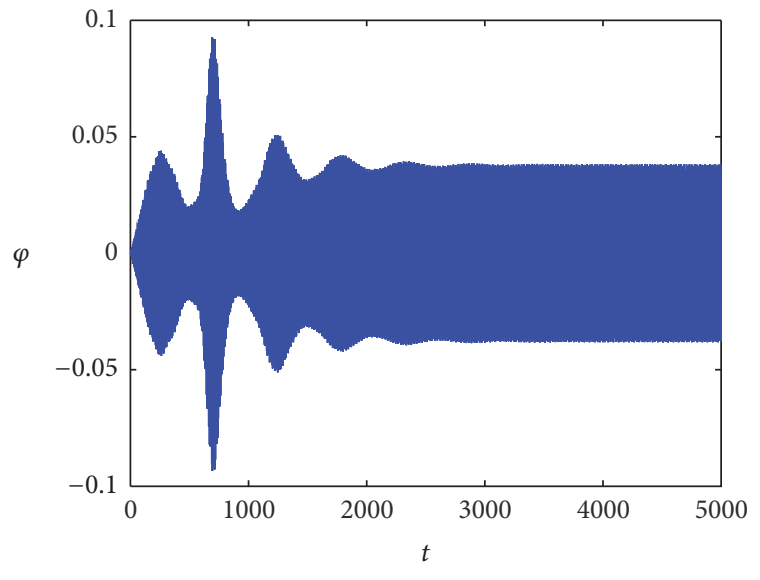

$-\sigma_{1}=0.01, \sigma_{2}=0.005$

(b)

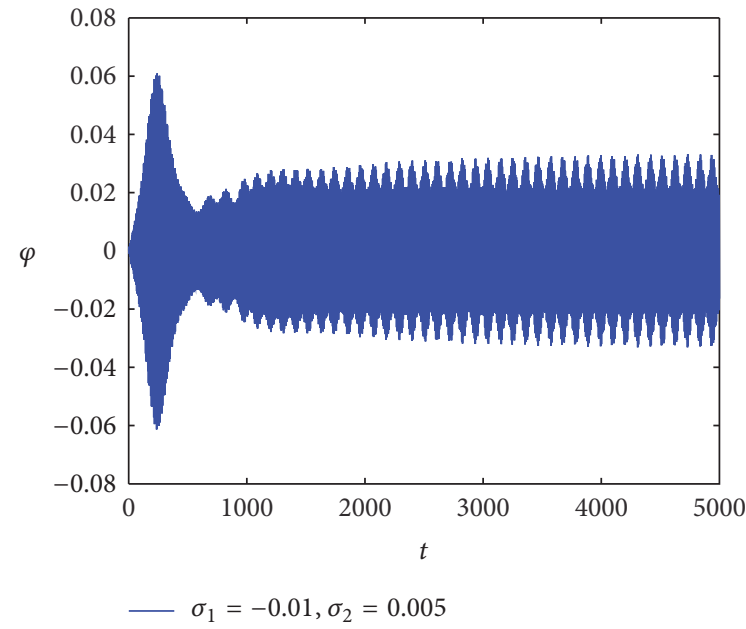

(d)

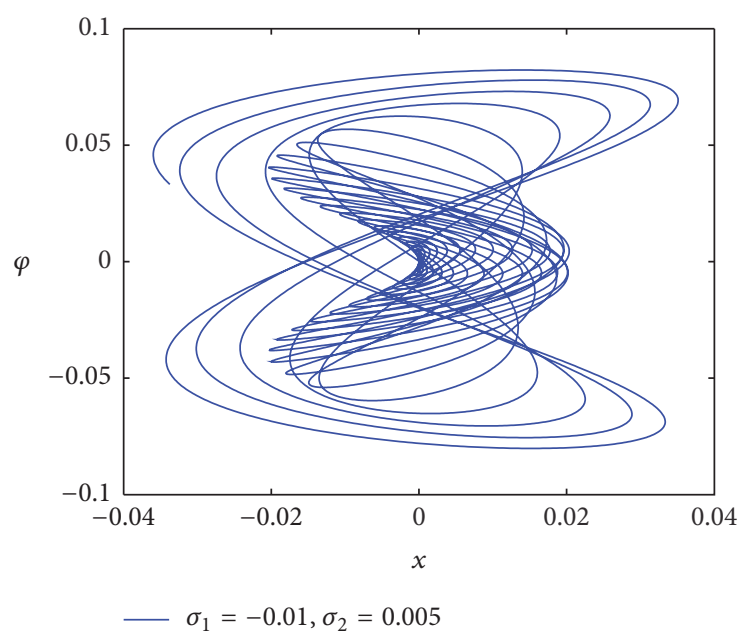

(f)

Figure 13: Parts (a), (b), (c), and (d) represent the portraits of time history, while parts (e) and (f) symbolize the projections of the phase planes at $a=b=r$. 


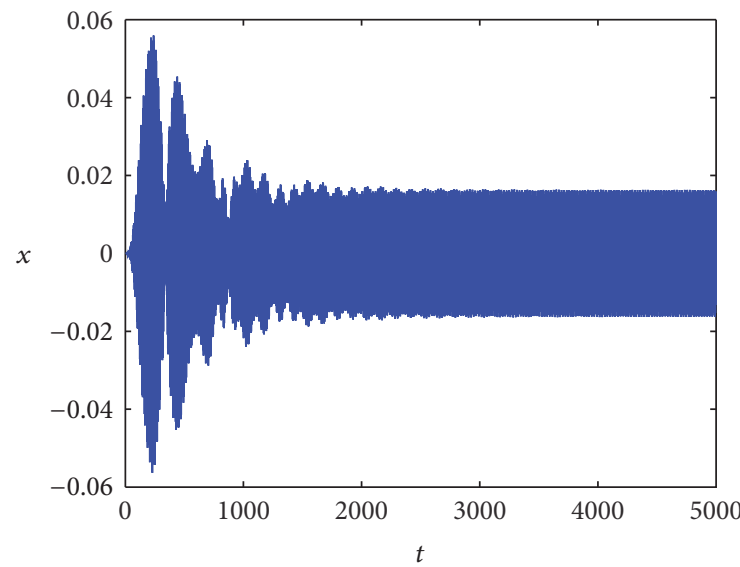

$-\sigma_{1}=0.01, \sigma_{2}=0.005$

(a)

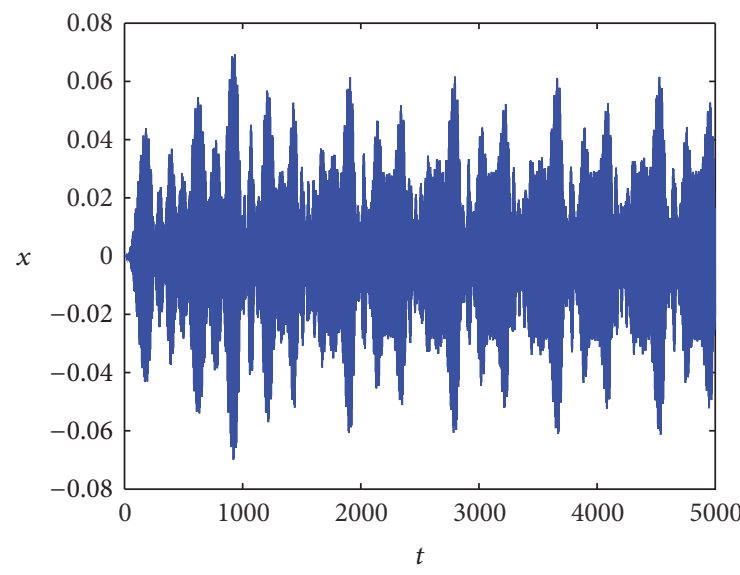

$-\sigma_{1}=-0.01, \sigma_{2}=0.005$

(c)

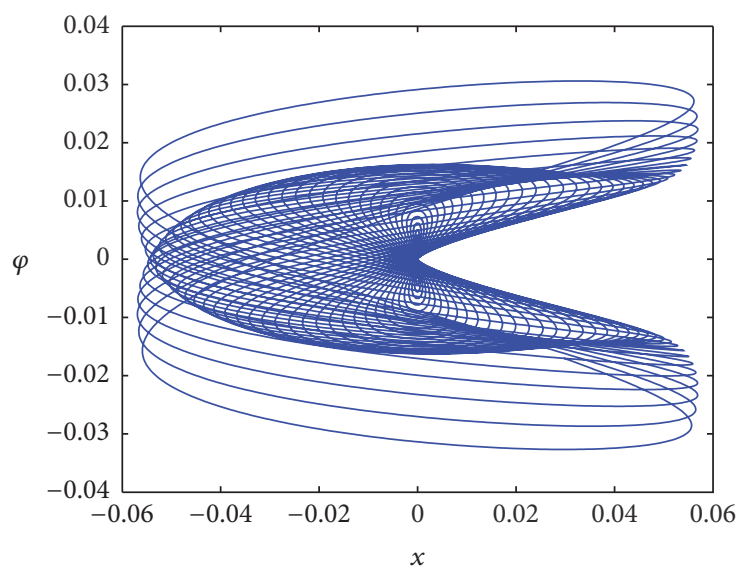

$-\sigma_{1}=0.01, \sigma_{2}=0.005$

(e)

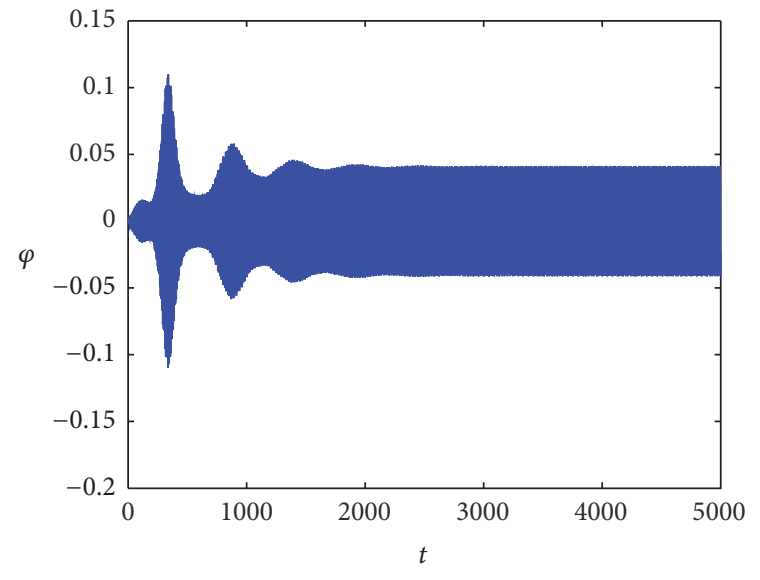

$-\sigma_{1}=0.01, \sigma_{2}=0.005$

(b)

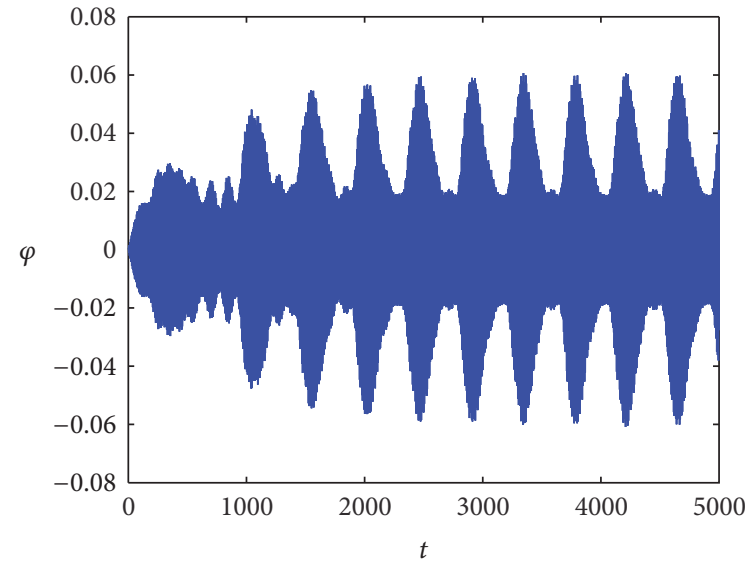

$-\sigma_{1}=-0.01, \sigma_{2}=0.005$

(d)

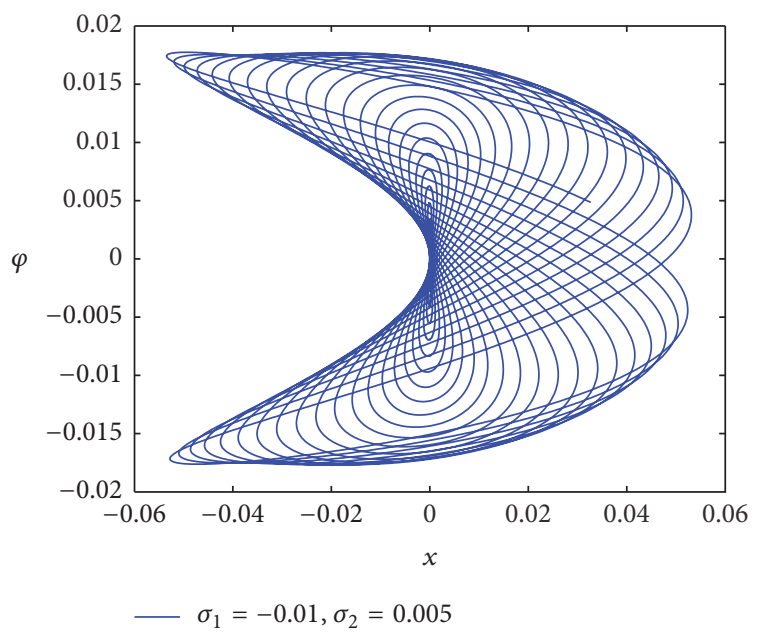

(f)

Figure 14: Plots (a), (b), (c), and (d) preform the portraits of time history, while plots (e) and (f) display the projections of the phase plane when $a=b=0$. 


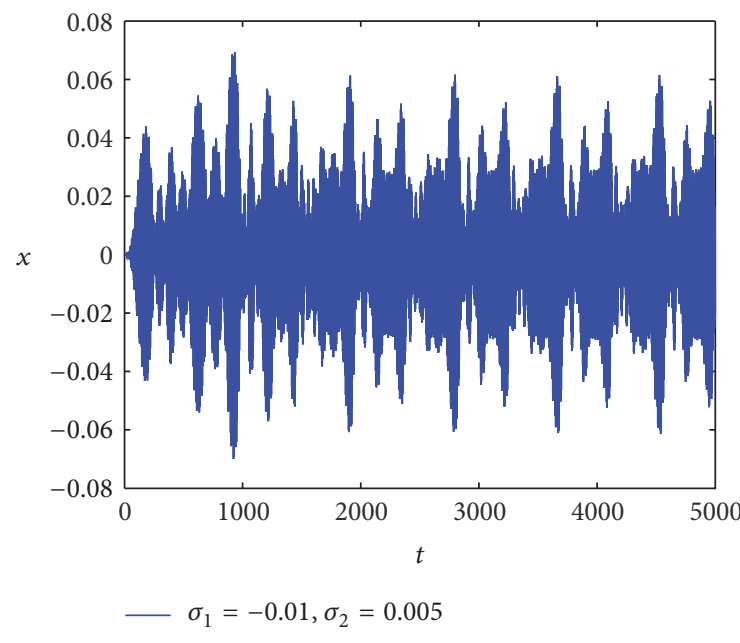

(a)

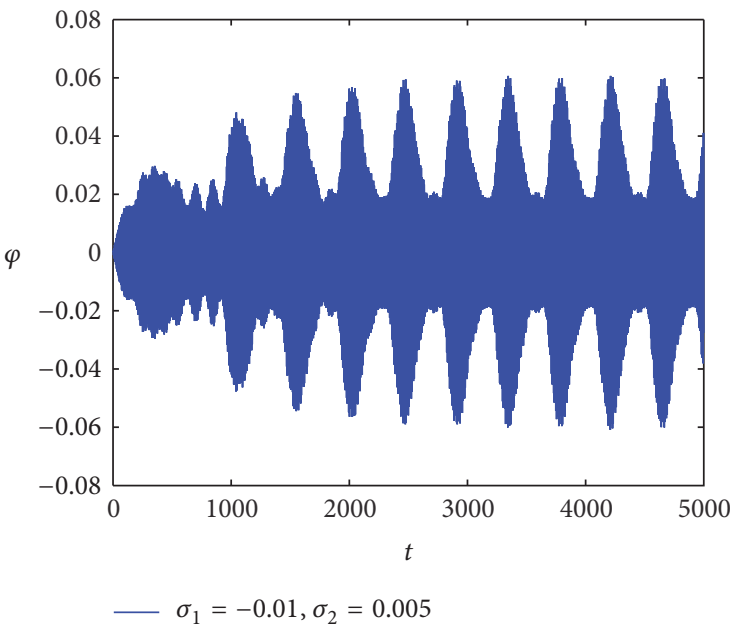

(b)

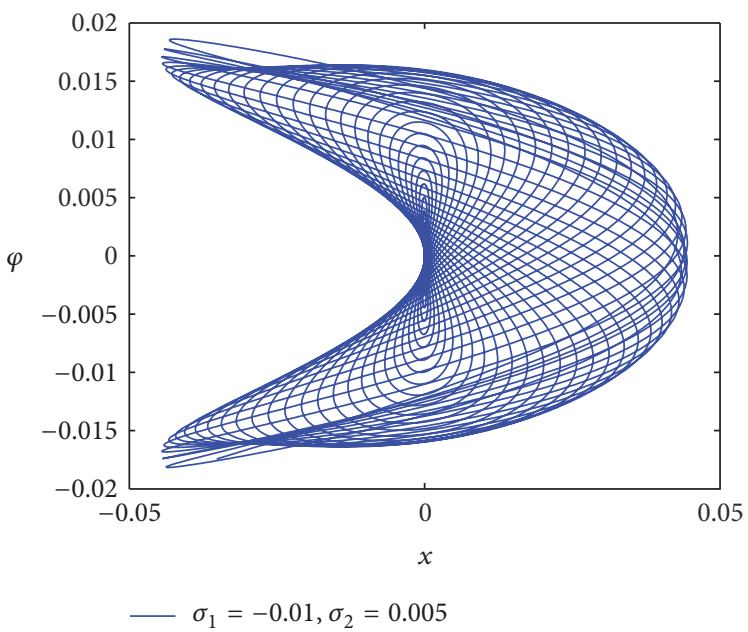

(c)

Figure 15: Parts (a) and (b) show the portraits of time history, while part (c) represents the variation of the solution $x$ versus the solution $\varphi$ when $a=0$.

movement of the nonlinear pendulum during time and to show the variation of the considered solutions with each other like parts (a) and (b) of Figure 16 for the time history evaluation and part (c) for the variation of the attained solutions. These plots are calculated during the period of time from 0 to $500 \mathrm{~s}$ at $\sigma_{1}=0.01\left[\mathrm{rad} \cdot \mathrm{s}^{-1}\right], \sigma_{2}=0.005\left[\mathrm{rad} \cdot \mathrm{s}^{-1}\right]$, $\omega_{1}=1\left[\mathrm{rad} \cdot \mathrm{s}^{-1}\right], \omega_{2}=0.5\left[\mathrm{rad} \cdot \mathrm{s}^{-1}\right]$, and $a=0.3[\mathrm{~m}]$.

It must be notice that the steady-state solutions at $t=0$ are trivial ones as shown from parts (a) and (b) from this figure.

\section{Conclusion}

The analytical solutions of the derived original system (represents a nonlinear 2-DOF equations (5)) up to the third approximation are obtained using the MS method. The determination of all possible resonances that might occur is presented. One of these possible resonances, namely, the principal external resonances when $\Omega \simeq \omega_{1}$ and $\Omega_{2} \simeq \omega_{2}$, is studied. Moreover, the modulation equations are obtained and solved numerically. The possible amplitudes have been deduced in (26) and (27) for the parametric and for the external resonances, respectively. These amplitudes are represented graphically through different plots. The substantial conditions for the stability analysis of the investigated system have been achieved according to Routh-Hurwitz method in order to obtain the possible fixed points. The projections of the phase plane of both solutions $x$ and $\varphi$ are represented graphically under certain parameters to show the effect of the different parameters on the motion. Some special cases from this work are presented in order to simulate the dynamic behavior of the considered model. Therefore, the present results are in high consistency with the known previous results as in [17-20]. One of the important applications of the considered model is the treatment of the seismic waves for the ground vibrations caused by seismic sources like earthquakes and volcano eruptions.

\section{Competing Interests}

The authors declare that they have no competing interests. 


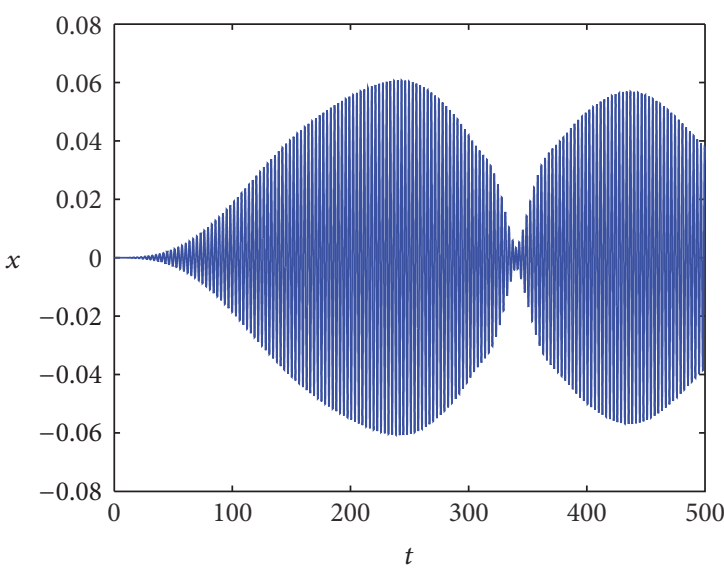

$-\sigma_{1}=0.01, \sigma_{2}=0.005$

(a)

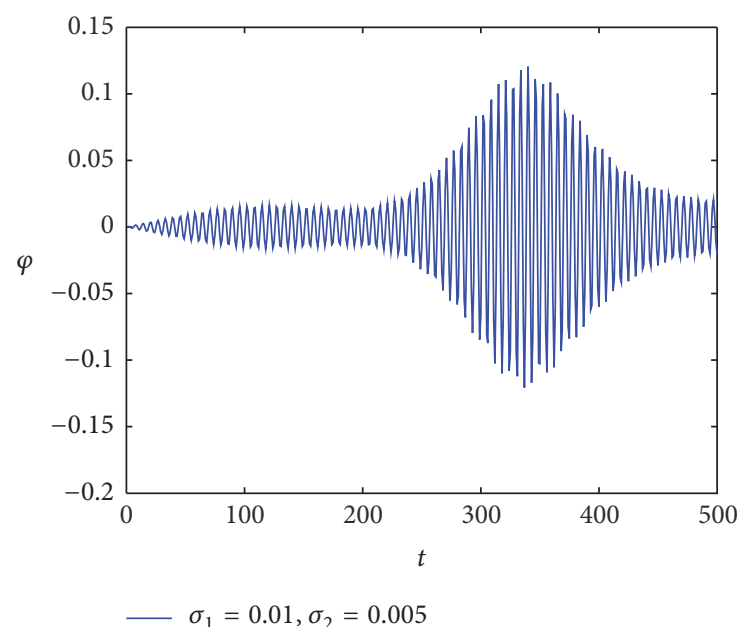

(b)

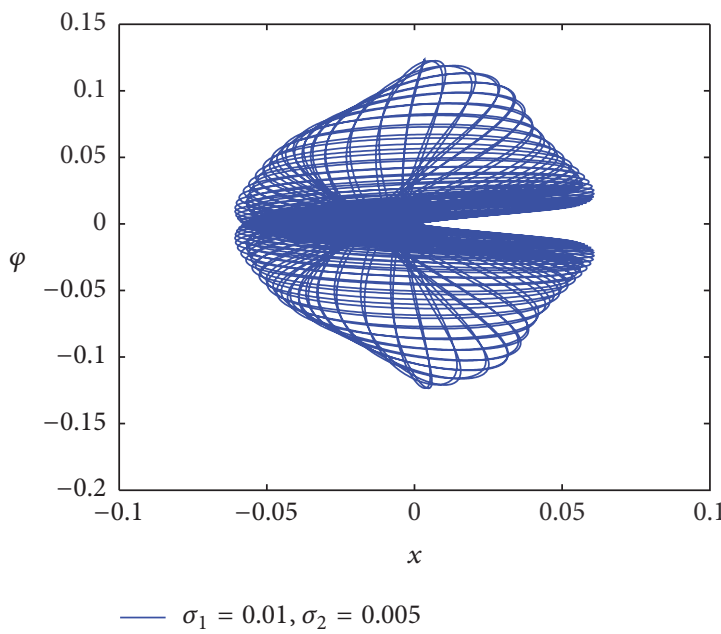

(c)

FIGURE 16: Parts (a) and (b) illustrate the portraits of time history, while part (c) displays the variation of the attained solutions $x$ and $\varphi$ when $b=0$.

\section{References}

[1] J. Miles, "Resonant motion of a spherical pendulum," Physica D: Nonlinear Phenomena, vol. 11, no. 3, pp. 309-323, 1984.

[2] J. Miles, "Resonantly forced motion of two quadratically coupled oscillators," Physica D. Nonlinear Phenomena, vol. 13, no. 1-2, pp. 247-260, 1984.

[3] S. Tousi and A. K. Bajaj, "Period-doubling bifurcations and modulated motions in forced mechanical systems," ASME Journal of Applied Mechanics, vol. 52, no. 2, pp. 446-452, 1985.

[4] A. Maewal, "Chaos in a harmonically excited elastic beam," Journal of Applied Mechanics, vol. 53, no. 3, pp. 625-632, 1986.

[5] A. H. Nayfeh, "Parametric excitation of two internally resonant oscillators," Journal of Sound and Vibration, vol. 119, no. 1, pp. 95-109, 1987.

[6] A. M. Abou-Rayan, A. H. Nayfeh, D. T. Mook, and M. A. Nayfeh, "Nonlinear response of a parametrically excited buckled beam," Nonlinear Dynamics, vol. 4, no. 5, pp. 499-525, 1993.

[7] A. K. Bajaj and S. Tousi, "Torus doublings and chaotic amplitude modulations in a two-degree-of-freedom resonantly forced mechanical system," International Journal of Non-Linear Mechanics, vol. 25, no. 6, pp. 625-641, 1990.

[8] W. K. Lee and H. D. Park, "Chaotic dynamics of a harmonically excited spring-pendulum system with internal resonance," Nonlinear Dynamics, vol. 14, no. 3, pp. 211-229, 1997.

[9] W. K. Lee and H. D. Park, "Second-order approximation for chaotic responses of a harmonically excited spring-pendulum system," International Journal of Non-Linear Mechanics, vol. 34, no. 4, pp. 749-757, 1999.

[10] M. Eissa, S. A. El-Serafi, M. El-Sheikh, and M. Sayed, "Stability and primary simultaneous resonance of harmonically excited non-linear spring pendulum system," Applied Mathematics and Computation, vol. 145, no. 2-3, pp. 421-442, 2003.

[11] A. Alasty and R. Shabani, "Chaotic motions and fractal basin boundaries in spring-pendulum system," Nonlinear Analysis: Real World Applications, vol. 7, no. 1, pp. 81-95, 2006.

[12] S. J. Zhu, Y. F. Zheng, and Y. M. Fu, "Analysis of non-linear dynamics of a two-degree-of-freedom vibration system with non-linear damping and non-linear spring," Journal of Sound and Vibration, vol. 271, no. 1-2, pp. 15-24, 2004. 
[13] T. S. Amer and M. A. Bek, "Chaotic responses of a harmonically excited spring pendulum moving in circular path," Nonlinear Analysis: Real World Applications, vol. 10, no. 5, pp. 3196-3202, 2009.

[14] R. Starosta, G. Sypniewska-Kamińska, and J. Awrejcewicz, "Asymptotic analysis of kinematically excited dynamical systems near resonances," Nonlinear Dynamics, vol. 68, no. 4, pp. 459-469, 2012.

[15] J. Awrejcewicz, R. Starosta, and G. S. Kaminska, "Asymptotic analysis of resonances in nonlinear vibrations of the 3-dof pendulum," Differential Equations and Dynamical Systems, vol. 21, no. 1-2, pp. 123-140, 2013.

[16] F. R. Gantmacher, Applications of the Theory of Matrices, John Wiley \& Sons, New York, NY, USA, 2005.

[17] R. Starosta and J. Awrejcewicz, "Asymptotic analysis of parametrically excited spring pendulum," in SYROM 2009, I. Visa, Ed., pp. 421-432, Springer, Berlin, Germany, 2009.

[18] R. Starosta, G. S. Kaminska, and J. Awrejcewicz, "Parametric and external resonances in kinematically and externally excited nonlinear spring pendulum," International Journal of Bifurcation and Chaos, vol. 21, no. 10, pp. 3013-3021, 2011.

[19] J. Awrejcewicz and R. Starosta, "Resonances in a kinematically driven nonlinear system-Asymptotic analysis," MESA, vol. 1, no. 1, pp. 1-10, 2010.

[20] J. Awrejcewicz, R. Starosta, and G. Sypniewska-Kamińska, "Asymptotic analysis and limiting phase trajectories in the dynamics of spring pendulum," in Applied Non-Linear Dynamical Systems, vol. 93 of Springer Proceedings in Mathematics \& Statistics, pp. 161-173, Springer, 2014. 


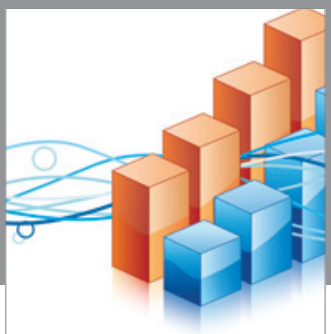

Advances in

Operations Research

vatem alat4

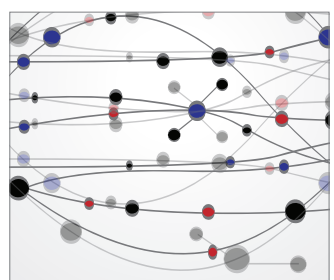

\section{The Scientific} World Journal
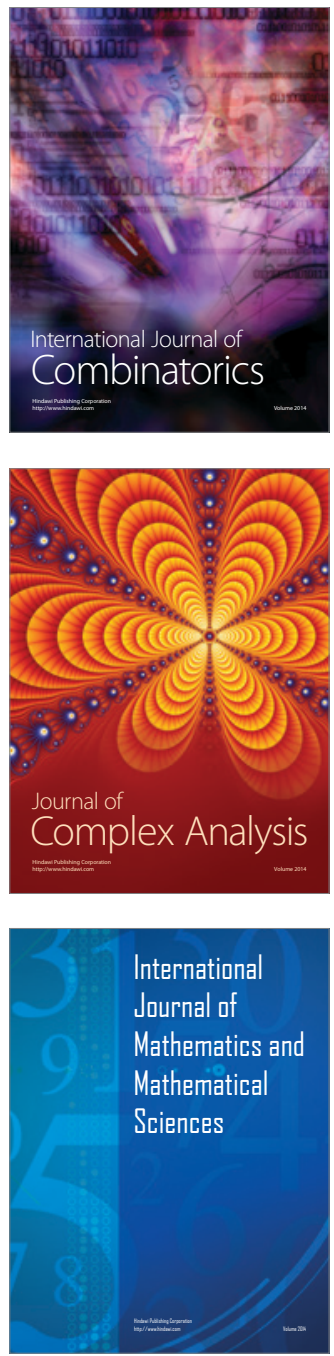
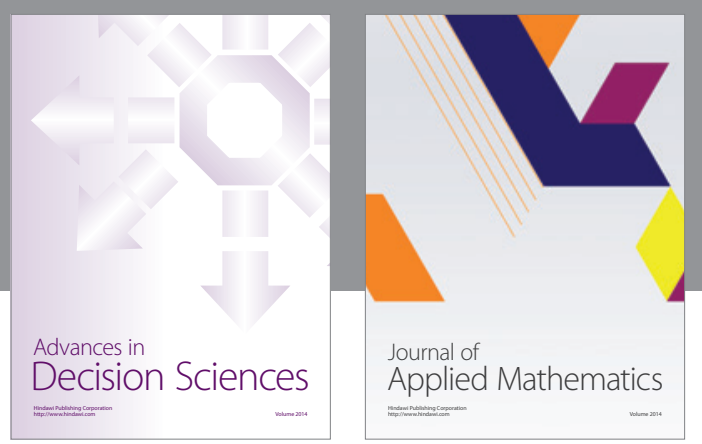

Algebra

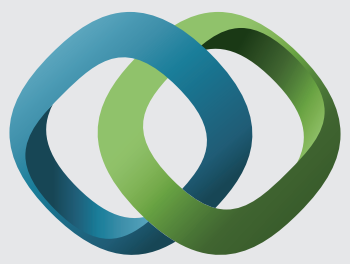

\section{Hindawi}

Submit your manuscripts at

http://www.hindawi.com
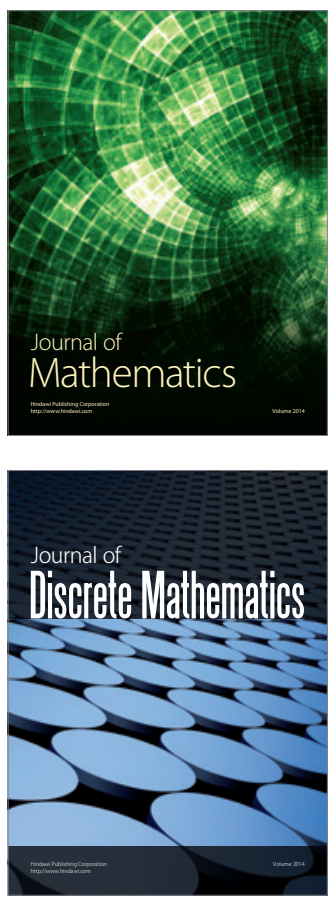

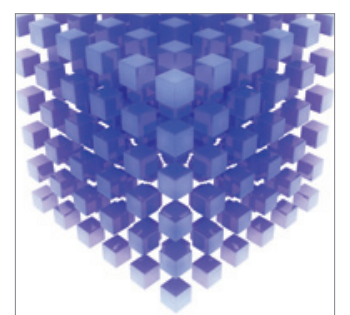

Mathematical Problems in Engineering
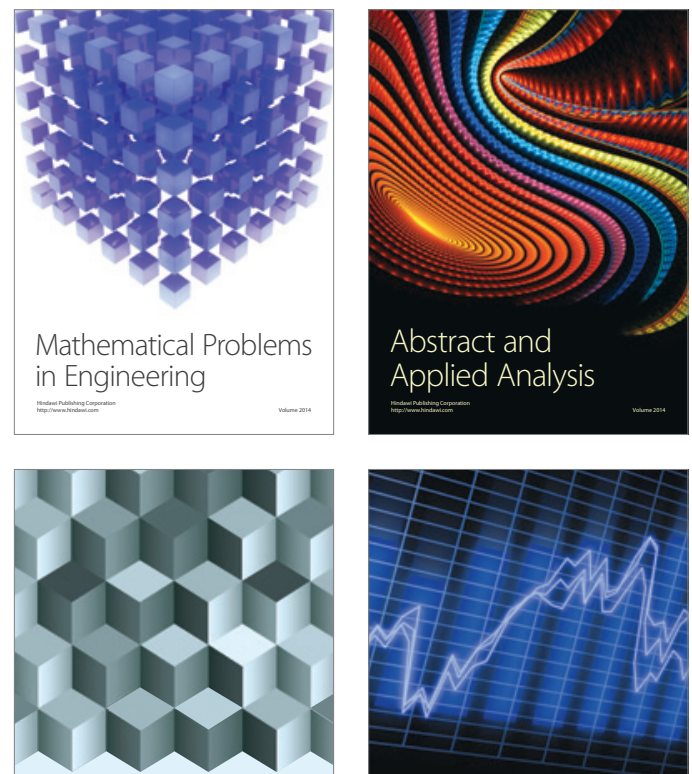

Journal of

Function Spaces

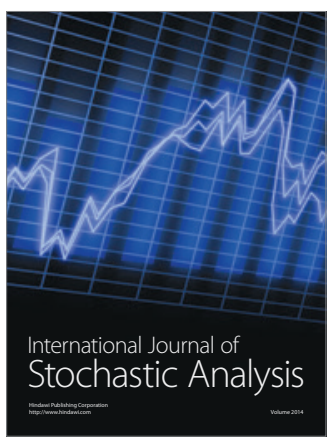

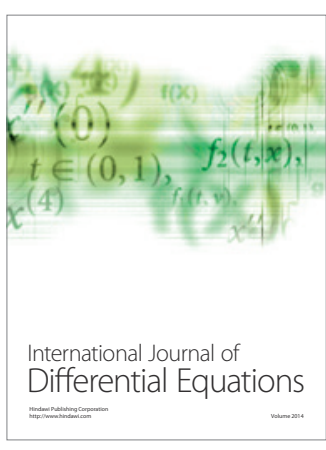
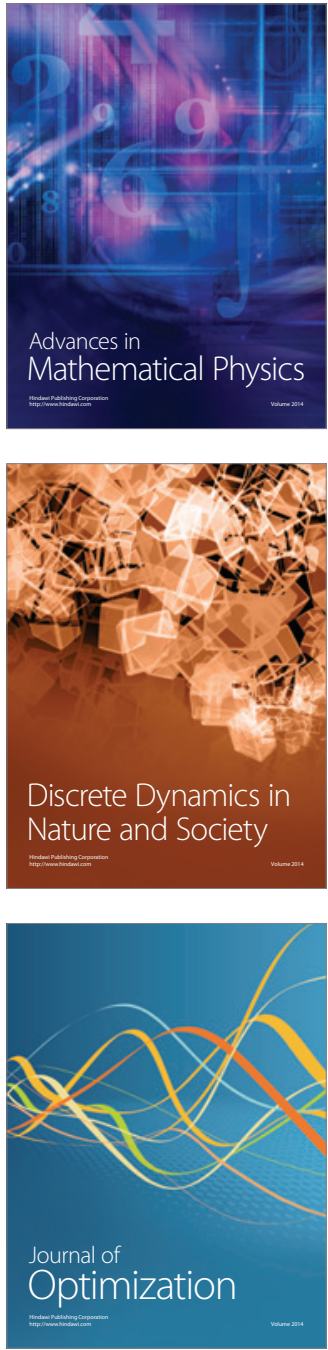